\title{
CLASSIFICATION ET CHRONOLOGIE DE BAS FOURNEAUX DE RÉDUCTION DU FER À USAGE UNIQUE AU SUD-OUEST DU NIGER
}

Rodrigue Guillon, C. Petit, Jean L. Rajot, A. Abdourhamane Touré, Oumarou A. Idé, Z. Garba

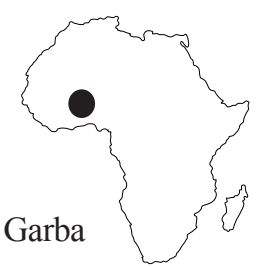

Résumé

Au sud-ouest du Niger dans les environs de Niamey, plusieurs milliers de bas fourneaux à usage unique ont été recensés. Une étude archéologique menée sur une trentaine de structures de bas fourneaux et de leurs scories a révélé la présence de quatre procédés de réduction du fer : trois de type scorie piégée et un de type scorie coulée. Les bas fourneaux à scorie piégée se différencient nettement par la morphologie et le volume de leur cuve. Les bas fourneaux à scorie coulée ont tous la particularité d'évacuer la scorie par de petits orifices creusés dans la cuve. L'écoulement de la scorie à l'extérieur de la cuve se fait soit verticalement soit latéralement. Selon les datations radiocarbone, l'activité métallurgique se développe dès le $2^{e}$ siècle $A D$ et s'intensifie jusqu'au $14^{e}$ siècle. Au-delà, elle évolue et perdure jusqu'au milieu du $20^{e}$ siècle. La production de fer par ces bas fourneaux est relativement faible, elle est principalement destinée au marché local.

\begin{abstract}
In southwestern Niger, near Niamey, several thousand singleuse bloomery furnaces have been mapped and identified. The archaeological study of approximately 30 furnaces and their slag reveals the existence of four methods for iron smelting: three types of pit furnace and one slag-tapping type. The slag pit furnaces are clearly differentiated by the form and volume of their pits. All slag-tapping furnaces drain off slag through small openings. The slag is tapped either vertically or laterally. According to radiocarbon dates, the smelting activity developed in the $2^{\text {nd }}$ century $A D$ and intensified through to the $14^{\text {th }}$ century. It continued to evolve until the middle of the $20^{\text {th }}$ century. The low intensity of iron production for these furnaces indicates the products were intended mainly for the local market.
\end{abstract}

Mots clés: Bas fourneaux, métallurgie du fer, sahel, scorie piégée, scorie coulée

Rodrigue Guillon $\}$ rodrigue.guillon@mae.u-paris10.fr / C.Petit

$\triangle$ UMR 7041 ArScAn, Équipe “Archéologies environnementales”, Nanterre, France

J.L. Rajot

first names to be written out - please provide $\triangle \quad$ UMR IRD 242 iEES, Paris, France

and IRA, Médenine, Tunisie

\section{A. Abdourhamane Touré / Z. Garba}

$\triangle$ Département de géologie, UAM, Niamey, Niger

Oumarou A. Idé

$\triangle \quad$ IRSH, Niamey, Niger 


\section{Introduction}

En Afrique, les bas fourneaux de réduction du fer montrent une grande diversité tant de formes architecturales que de procédés de réduction du fer. (CLINE 1937 ; Francis-Boeuf 1937 ; Pole 1985 ; Sutton 1985 ; MaRTINELli 1993 ; MCNAUGhton 1993). Cette métallurgie du fer est, en Afrique subsaharienne, uniquement fondée sur la méthode directe de réduction. Elle repose sur la transformation du minerai de fer en fer-métal, plus ou moins aciéré, dans un bas fourneau. Les oxydes de fer contenus dans le minerai sont réduits par le monoxyde de carbone produit par la combustion du charbon de bois (LEROY 2001). L'éponge de fer issue de cette réduction est un bloc de métal contenant de nombreuses poches de gaz et d'impuretés. Elle doit être épurée par martelage à chaud avant d'être forgée. Le résidu de la fusion partielle du minerai de fer est la scorie de réduction.

Pour le Sud-ouest du Niger, les plus anciens indices d'activité métallurgique auraient été obtenus dans la partie septentrionale vers 500 cal BC (VERNET 1996). Plus à l'est, l'ancienneté de l'activité métallurgique est encore largement discutée (AlPERn 2005 ; Clist 2012).

Dans la région de Niamey, un très grand nombre de blocs de scories et de cuves de bas fourneaux ont été cartographiés (GuILlon et al. 2012). La présence de blocs isolés et de fragments de scorie, l'abondance des structures en terre cuite mélangées aux scories et l'absence d'amoncellement témoignent de vestiges de bas fourneaux à usage unique. Cette pratique qui consiste à construire un bas fourneau pour une unique opération de réduction est connue dans la région soudano-sahélienne ouest-africaine. Elle a été identifiée sur des vestiges archéologiques dans la vallée du Sénégal (RoBERT-CHALEIX \& Sognane 1983 ; Robert-Chaleix 1994 ; McIntosh \& Bocoum 2000 ; Killick 2015), au Mali (ChiÈze 1991), au Burkina Faso (KIENON-KabORÉ 2005 ; KIETHÉGA 2006 ; FABre 2009 ; SERnEels et al. 2012, 2014) et au nord Bénin (RoBION-BRUNNER et al. 2015). D'un point de vue ethnographique, ce procédé a également été étudié auprès de forgerons en activité au début du $20^{\mathrm{e}}$ siècle, dans la région de l'Ader au sud de Tahoua au Niger (ÉCHARD 1968,1983 , 1986) et à Banizoumbou à $80 \mathrm{~km}$ à l'est de Niamey en 1998 (com. pers. J.L. Rajot).

L'objectif de cet article est de proposer une classification des bas-fourneaux à usage unique de la région de Niamey en s'appuyant sur l'étude détaillée de 36 structures issues d'un corpus archéologique de plus de 10000 unités. La description typologique à partir des éléments architecturaux et des scories conservées nous a conduits à formuler des hypothèses de fonctionnement. Nous présenterons ensuite les datations radiocarbone et nous replacerons nos résultats dans le contexte régional.

\section{Zone d'étude}

La zone d'étude se situe entre la vallée du fleuve Niger et le Dallol Bosso, affluent fossile du fleuve. Cette région actuellement en domaine climatique soudano-sahélien est marquée par la présence de plateaux cuirassés qui atteignent pour les plus hauts $50 \mathrm{~m}$ au-dessus des talwegs. Des bancs d'oolithes ferrugineuses affleurent sur les talus de ces plateaux. Les vallées sableuses sont parcourues par des ravines dont l'écoulement est temporaire. Cinq secteurs géographiques ont été étudiés. Le principal est localisé à $15 \mathrm{~km}$ à l'est de Niamey dans le bassin versant des lacs de Saga Gorou. Deux autres sont situés, à $60 \mathrm{~km}$ plus à l'est dans les environs du village de Banizoumbou et sur la bordure occidentale du Dallol Bosso près de Gobéri. En rive gauche, les recherches se sont concentrées sur les plateaux et la vallée de Louguel et sur la rive gauche du Goroubi à $65 \mathrm{~km}$ au sud de Niamey (Fig. 1).

\section{Prospections pédestres et sondages archéologiques}

Les bas fourneaux ont été cartographiés et repérés au cours de prospections pédestres. Ils sont aisément repérables dans le paysage steppique sahélien par la présence de blocs ou de fragments sombres de scorie et/ ou d'éléments indurés argilo-sableux d'une teinte brunorangé provenant du démantèlement de la superstructure. Les bas fourneaux sont identifiables soit par des amoncellements de fragments de scorie sous lesquels affleurent les contours d'une cuve argilo-sableuse qui couvre une surface d'environ un mètre de diamètre, soit par des blocs de scorie isolés (Fig. 2).

Après identification des scories et des structures en terre cuite, 21 sondages archéologiques ont été réalisés dans les différents secteurs afin de comprendre l'architecture et le fonctionnement des bas fourneaux.

\section{Corpus archéologique}

Sur l'ensemble des zones prospectées, 10175 bas fourneaux ont été recensés. Ils sont répartis sur quatre zones (Fig. 1) :

- 4938 pour la région des trois lacs de Saga Gorou dont 4504 dans les limites de leur bassin versant, parmi eux 27 ont été étudiés ;

- 1017 dans la vallée de Louguel, trois ont été fouillés ; 1612 autour du village de Banizoumbou, trois ont été sondés ;

- 2608 le long de la bordure ouest du plateau du Dallol Bosso près de Gobéri où un seul bas fourneau a été étudié. 


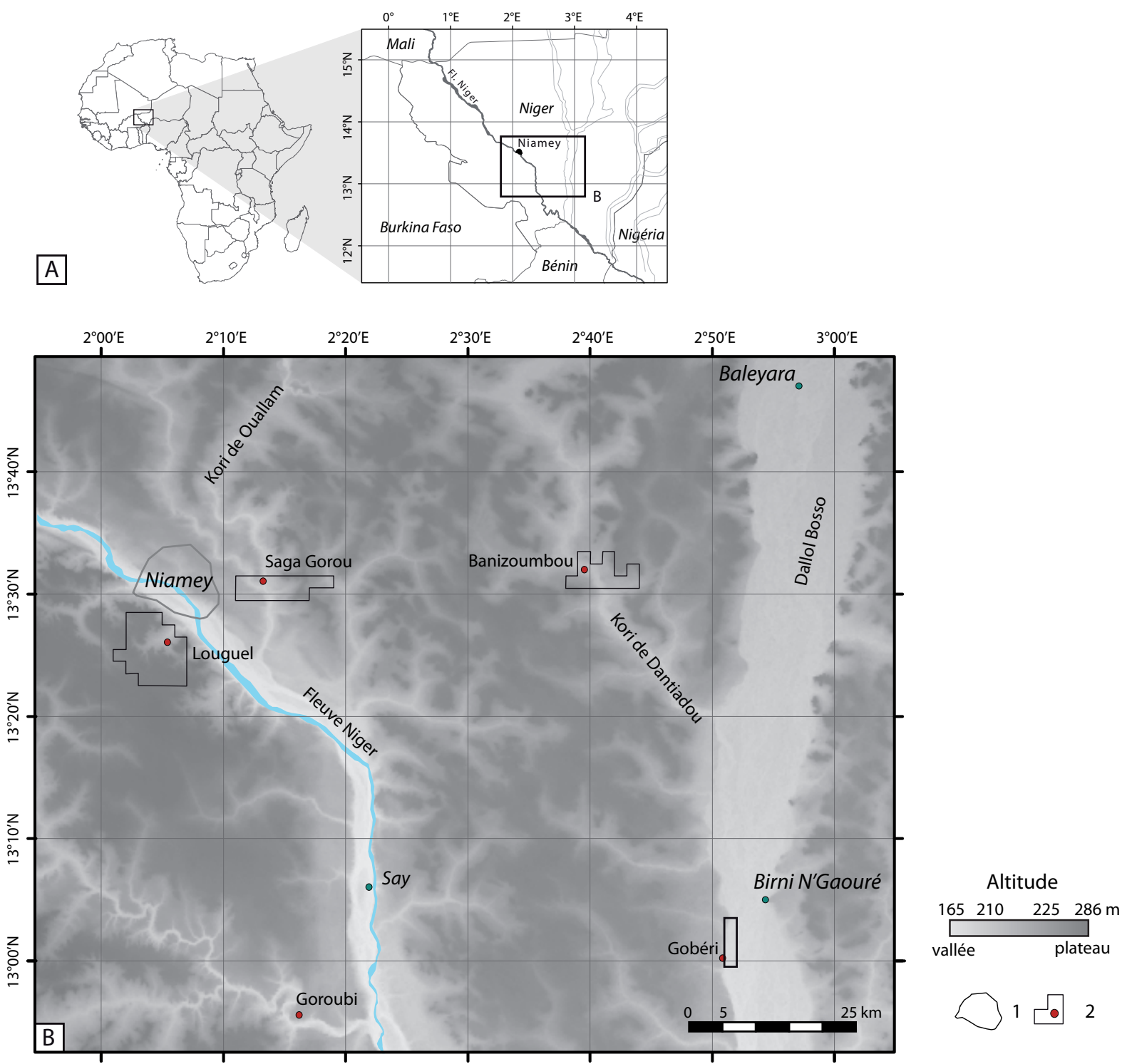

Figure 1. Carte de localisation des secteurs prospectés. A : Afrique et le Sud-ouest du Niger ; B : Zone d'étude entre la vallée du fleuve Niger et le Dallol Bosso ; 1 : Zone urbaine de Niamey ; 2 : Zone de prospection.
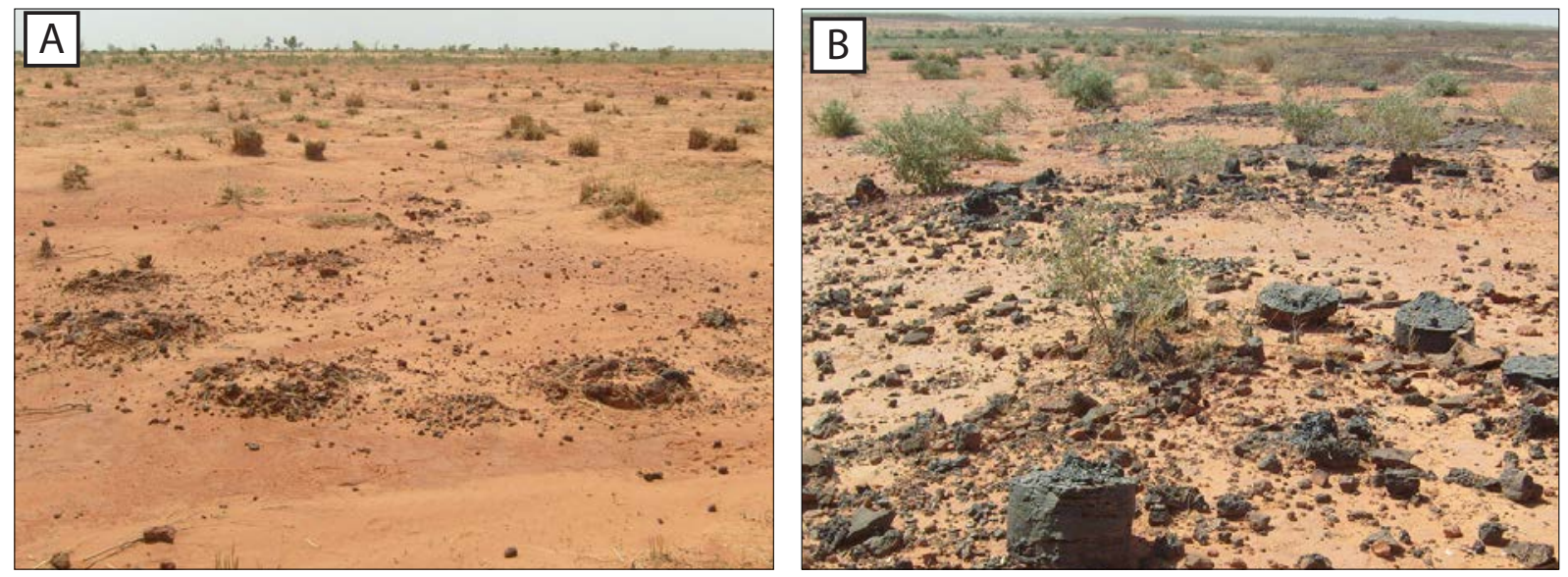

Figure 2. Vestiges de bas fourneaux. A : Amas circulaires constitués de fragments de scorie et de terre cuite sur des sols non érodés ; B : Blocs massifs de scorie sur des sols érodés. 
Le secteur de Goroubi n'a pas été prospecté, toutefois un bas fourneau a été fouillé.

Le minerai communément utilisé provient des bancs d'oolithes ferrugineuses affleurant dans le talus des plateaux. Concassé en blocs de quelques centimètres, ce minerai a été plusieurs fois retrouvé amoncelé à proximité de différents bas fourneaux.

\section{Datation}

L'ensemble des datations est issu d'analyses radiocarbone sur des charbons de bois collectés lors des différents sondages archéologiques. 43 dates radiocarbone ont été réalisées, 31 ont été retenues (Tab. 1 ; Figs. 17 et 18). Les datations les plus fiables ont été obtenues sur des charbons de bois situés dans le remblai de la cuve ou mieux encore piégés dans la scorie, cependant ces conditions sont peu fréquentes. Ainsi douze datations ont été rejetées, dix charbons de bois dont la position stratigraphique était déconnectée des structures archéologiques ont été considérés comme trop anciens par rapport à l'ensemble. Deux autres trop jeunes, polluaient manifestement des structures plus anciennes.

\section{Classification des bas fourneaux}

Une première distinction fondamentale dans la classification des bas fourneaux concerne la possibilité de réutiliser ou non la structure de réduction. Elle conduit à distinguer les bas fourneaux à usage multiple des bas fourneaux à usage unique. Sur l'ensemble du corpus disponible, seules trois structures correspondent à des bas fourneaux à usage multiple. Même si aucun sondage n'a été mené sur ces structures, une datation a été obtenue et une description sommaire peut être présentée.

\section{Bas fourneaux à usage multiple}

Les trois bas fourneaux à usage multiple (notés BFUM dans la classification) sont situés sur le plateau de Louguel. Ils sont constitués de structures en terre cuite à coté desquelles se concentrent des scories très fragmentées correspondant à une zone de rejet localisée au-devant de chaque bas fourneau. La base de la cheminée est conservée en place, l'épaisseur de la paroi est d'environ $15 \mathrm{~cm}$. Des évents sont clairement identifiables, ils correspondent à l'emplacement de

\begin{tabular}{|c|c|c|c|c|c|c|c|}
\hline Nom & Lieu & Type & Cote $(\mathrm{cm})$ & Fig. & $N^{\circ}$ labo & Date 14C BP & Date cal AD (2s) \\
\hline BNG08F1-2 & Gobéri & $\mathrm{SC}-\mathrm{V}$ & -- & -- & Ly-6149 & $1785 \pm 30$ & $133 / 335$ \\
\hline BNG08F1-1 & Gobéri & $\mathrm{SC}-\mathrm{V}$ & -- & -- & Ly-6148 & $1770 \pm 30$ & $137 / 345$ \\
\hline BFD08F1-3 & Saga Gorou & inconnu & -30 & -- & Ly-6311 & $1680 \pm 30$ & $258 / 425$ \\
\hline BFD08F4-A3-2 & Saga Gorou & $\mathrm{SC}-\mathrm{V}$ & -31 & 10 & Poz-39924 & $1675 \pm 30$ & $258 / 427$ \\
\hline GB08F2-5 & Saga Gorou & $\mathrm{SC}-\mathrm{V}$ & -71 & 9 & Ly-6321 & $1625 \pm 30$ & $352 / 537$ \\
\hline GB08F1-7 & Saga Gorou & SC-V & -66 & 8 & Ly-6320 & $1600 \pm 30$ & $404 / 540$ \\
\hline BKW08F1-7 & Saga Gorou & $\mathrm{SPa}$ & -20 & 6 & Ly-6316 & $1480 \pm 30$ & $540 / 644$ \\
\hline Gbi09F1-2 & Goroubi & $\mathrm{SPa}$ & -- & 6 & Ly- 6328 & $1465 \pm 30$ & $551 / 646$ \\
\hline GB08F14-2 & Saga Gorou & $\mathrm{SPa}$ & -12 & -- & Ly-6333 & $1375 \pm 30$ & $607 / 685$ \\
\hline LG09F1-4 & Louguel & $\mathrm{SC}-\mathrm{V}$ & & 9 & Poz-39926 & $1330 \pm 30$ & $647 / 770$ \\
\hline B08F16-2 & Saga Gorou & $\mathrm{SC}-\mathrm{V}$ & -10 & -- & Ly-6335 & $1310 \pm 30$ & $656 / 773$ \\
\hline GB08F11-2 & Saga Gorou & SC-pv & -5 & -- & Ly-6331 & $1290 \pm 30$ & $663 / 775$ \\
\hline SG08F1-1 & Saga Gorou & SPff & -4 & 4 & Ly-6141 & $1270 \pm 30$ & $664 / 855$ \\
\hline LG09F2-2/2 & Louguel & $\mathrm{SC}-\mathrm{V}$ & -- & -- & Poz-41637 & $1260 \pm 30$ & $669 / 862$ \\
\hline LG09F2-2/1 & Louguel & SC-V & -- & -- & Poz-39929 & $1245 \pm 30$ & $682 / 870$ \\
\hline SG08F1-5 & Saga Gorou & SPff & -50 & 4 & Ly-6145 & $1245 \pm 30$ & $682 / 870$ \\
\hline SG08F1-2 & Saga Gorou & SPff & -9 & 4 & Ly-6142 & $1235 \pm 30$ & $687 / 879$ \\
\hline GB08F15-1 & Saga Gorou & SC-V & -25 & -- & Ly-6334 & $1225 \pm 30$ & $690 / 885$ \\
\hline SG08F3-3 & Saga Gorou & SPff & -22 & 4 & Ly-6338 & $1220 \pm 30$ & $692 / 888$ \\
\hline SG08F1-4 & Saga Gorou & SPff & -40 & 4 & Ly-6144 & $1215 \pm 30$ & $693 / 890$ \\
\hline SG08F1-3 & Saga Gorou & SPff & -20 & 4 & Ly-6143 & $1210 \pm 30$ & $694 / 892$ \\
\hline GB08F5-B3-1 & Saga Gorou & SC-1 & -15 & 12 & Ly-6324 & $1040 \pm 30$ & $898 / 1033$ \\
\hline GB08F5-B2-1 & Saga Gorou & SC-1 & -10 & 12 & Ly-6323 & $1035 \pm 30$ & $898 / 1036$ \\
\hline GB08F5-B4-1 & Saga Gorou & SC-1 & -20 & 12 & Ly-6325 & $1000 \pm 30$ & $982 / 1153$ \\
\hline BFD09F5-1 & Saga Gorou & SC-1 & -- & 12 & Ly-6317 & $930 \pm 30$ & $1025 / 1169$ \\
\hline GB08F6-4 & Saga Gorou & $\mathrm{SC}-\mathrm{V}$ & -24 & 9 & Ly-6326 & $855 \pm 30$ & $1051 / 1259$ \\
\hline GB08F6-5 & Saga Gorou & $\mathrm{SC}-\mathrm{V}$ & -50 & 9 & Ly-6327 & $840 \pm 30$ & $1058 / 1265$ \\
\hline GB08F9-5 & Saga Gorou & SC-1 & -23 & 13 & Ly-6329 & $705 \pm 30$ & $1259 / 1386$ \\
\hline PLRD09F1-1 & Louguel & BF-UM & scorie & 3 & Poz-39925 & $215 \pm 30$ & $1644 / 1955$ \\
\hline BNZ08F3-1 & Banizoumbou & SPff & -10 & -- & Ly-6319 & $175 \pm 30$ & $1656 / 1954$ \\
\hline BNZ08F2-1 & Banizoumbou & SPff & -10 & 5 & Ly-6318 & $140 \pm 30$ & $1669 / 1945$ \\
\hline
\end{tabular}

Tableau 1. Dates radiocarbone des bas fourneaux fouillés. 


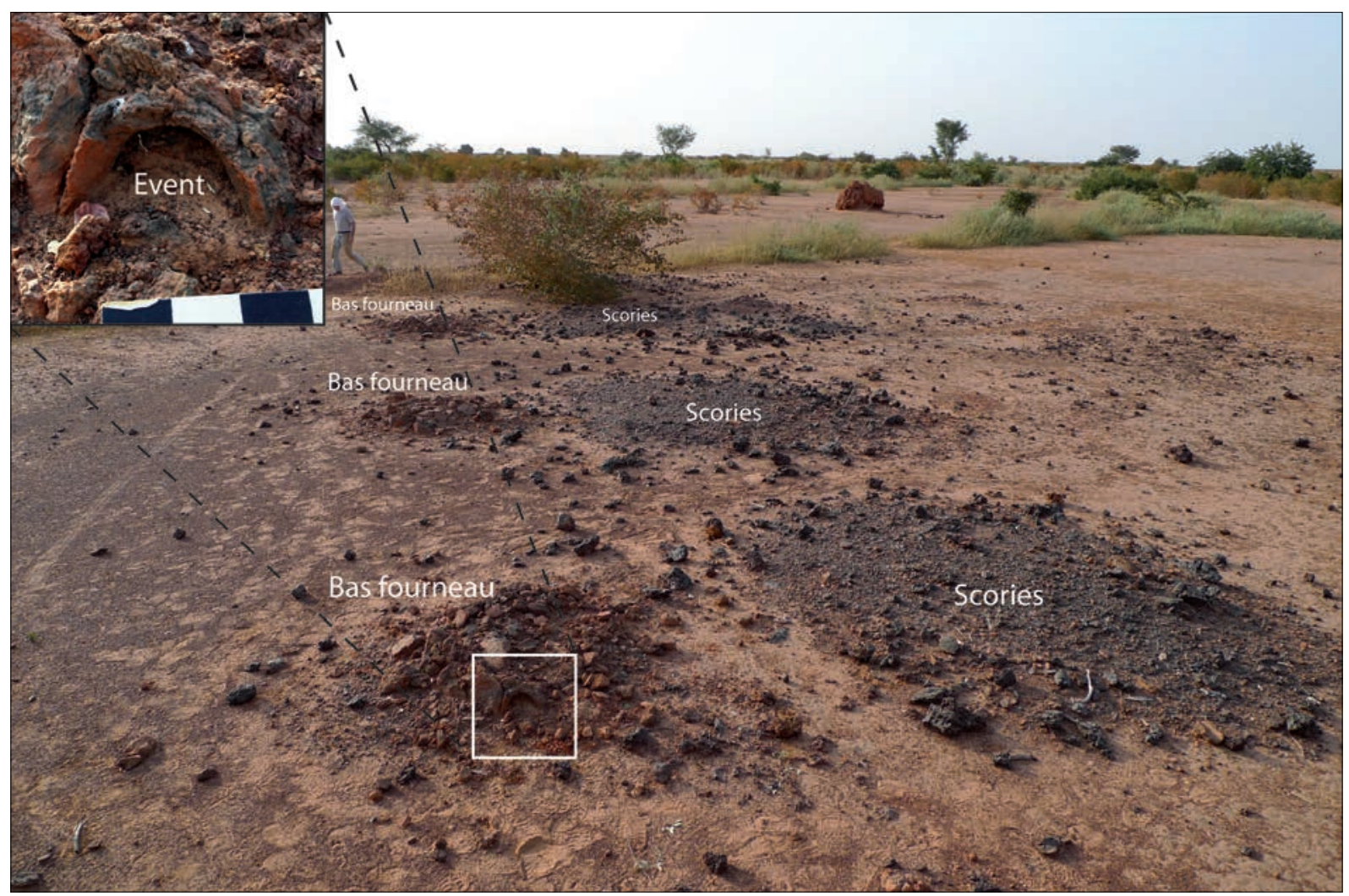

Figure 3. Bas fourneaux à usage multiple (BF-UM). La scorie est rejetée en dehors du bas fourneau. Détail sur un évent à la base de la cheminée.

tuyères amovibles qui suggèrent une ventilation naturelle (Fig. 3). La concentration des scories en dehors de la structure témoigne de l'évacuation intégrale des résidus de réduction pour le réemploi du bas fourneau ; le volume de scorie reste ici très limité et indique une utilisation de courte durée. Ces trois bas fourneaux peuvent être comparés à celui observé et reconstitué par Celis (1994) dans la région de Tillabéry (Niger) à $115 \mathrm{~km}$ au nord-ouest de Niamey. Ce type de bas fourneau présente toutefois une grande diversité de fonctionnement. Ils peuvent ainsi être à scorie piégée ou à scorie coulée latérale ou verticale (e.g., Celis 1987, 1994 ; ACKERMANN et al. 1999 ; Bellamy 2009). La comparaison peut difficilement être approfondie en raison de l'absence de sondage archéologique sur ces trois structures qui font figure d'exception dans la zone étudiée. Un charbon de bois piégé dans une scorie fournit une datation tardive. L'incertitude de la courbe de ${ }^{14} \mathrm{C}$ atmosphérique produit une marge d'erreur très large entre le milieu du $17^{\circ}$ et le milieu du $20^{\mathrm{e}}$ siècle. Cependant l'absence de témoignage par les populations locales et l'érosion des structures suggèrent qu'elles appartiendraient plutôt aux périodes anciennes de la fourchette de datation.

\section{Bas fourneaux à usage unique}

Ils représentent la quasi-totalité des structures étudiées et ont fait l'objet d'une trentaine de sondages archéologiques sur lesquels s'appuie la classification. Les éléments architecturaux en terre cuite constituant la superstructure des bas fourneaux à usage unique ne sont jamais conservés en place. La cheminée constituée de terre cuite avec un dégraissant végétal est relativement fragile. Non fondée dans le sol, elle repose vraisemblablement directement sur les tuyères. Ce procédé correspondrait aux cheminées amovibles et réutilisables attestées par les exemples ethnographiques (ÉCHARD 1965 , 1986). Les tuyères sont le plus souvent très fragmentées et semblent présenter peu de différences morphologiques. La cuve est l'élément architectural le mieux conservé quand le sol n'est pas érodé. Sa présence est toutefois trop aléatoire pour fonder une classification. Les seuls éléments abondants, omniprésents et de formes variées sur les vestiges d'un bas fourneau sont les scories. Nous proposons ainsi une classification typologique des bas fourneaux à usage unique à partir de la morphologie et de la position des scories au sein de structures de réduction encore en place. 
Notre classification des bas fourneaux s'appuie sur celle fondée sur la méthode de séparation de l'éponge de fer et de la scorie (CleERe 1972; TyleCOTE 1987 ; Pelet 1993 ; SERnEELs 1993, 1998 ; PLEINER 2000). Les scories constituées d'oxydes et de silicates, sont les déchets de la réduction du minerai de fer. Quand la température de fusion est atteinte dans le bas fourneau, l'éponge de fer se dissocie de la scorie. Trois grands types de bas fourneaux sont définis en fonction de la méthode de séparation entre le métal et la scorie : (i) la fosse, simple cuve sans séparation de l'éponge de fer et de la scorie, (ii) le bas fourneau à scorie piégée, où l'éponge de fer est au-dessus de la scorie qui est plus dense et reste piégée dans la cuve, (iii) le bas fourneau à scorie coulée, où l'éponge de fer est séparée de la scorie qui est évacuée en dehors de la cuve par une porte ou un orifice.

Dans notre cas, les bas fourneaux appartiennent au deux derniers types : les bas fourneaux de type scorie piégée (slag pit furnace) et ceux de type scorie coulée (slag-tapping furnace).

Trois types de bas fourneaux à scorie piégée avec un mode de piégeage bien différencié et un type de bas fourneaux à scorie coulée par un orifice présentant quatre variantes ont été identifiés.

\section{Bas fourneaux à Scorie Piégée en fond de four (SPff)}

À Saga Gorou, les bas fourneaux SG08F1 et SG08F3 (Fig. 4), sont constitués d'une cuve cylindrique à fond plat d'environ $80 \mathrm{~cm}$ de diamètre. Aucun indice de paroi n'est constaté, dans le substrat sableux, seules des zones de rubéfaction liées à l'échauffement du sol lors de la réduction sont visibles. Cette dépression est remplie de niveaux cendreux associés à des charbons de bois, de nombreux débris de terre cuite et des scories de toutes tailles. Leur surface est très rugueuse et les contours sont indéfinis même si certaines scories présentent des effets de paroi. Ces scories sont le plus souvent très poreuses, de nombreuses vacuoles témoignent soit d'anciennes bulles de dégazage, soit d'empreintes de matières périssables (charbons de bois, végétaux). Le contenu de ces cuves a été très perturbé par la récupération de l'éponge de fer ; après la réduction, la cuve est remblayée par des débris. Ces deux bas fourneaux sont construits sur les plateaux où le sol sableux est peu épais.

À Banizoumbou, le bas fourneau BNZ08F2 (Fig. 5), comme BNZ08F3, est similaire aux deux précédents, cependant plusieurs éléments les différencient. La cuve est de forme hémisphérique et constituée d'un épais enduit argilo-sableux. Le volume de cendre est élevé et les scories sont peu nombreuses. De plus, ces bas fourneaux sont principalement construits sur les glacis sableux.
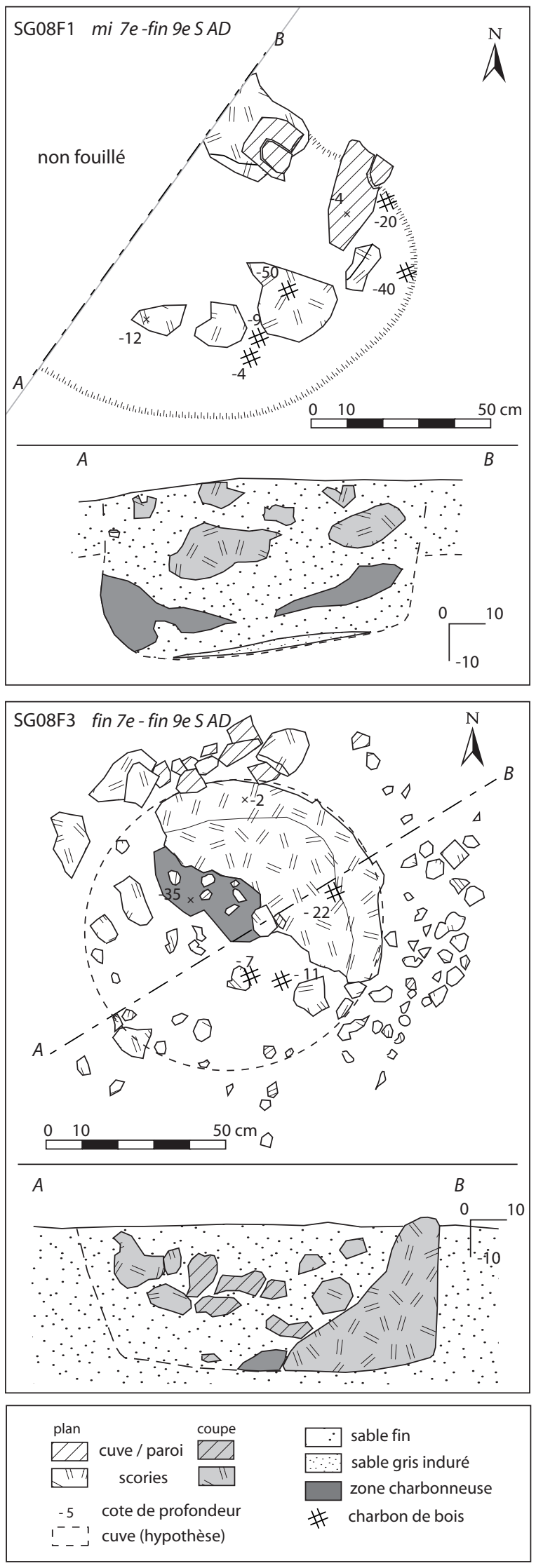

Figure 4. Plan et coupe des bas fourneaux à scorie piégée en fond de four (SPff) SG08F1 et SG08F3. 


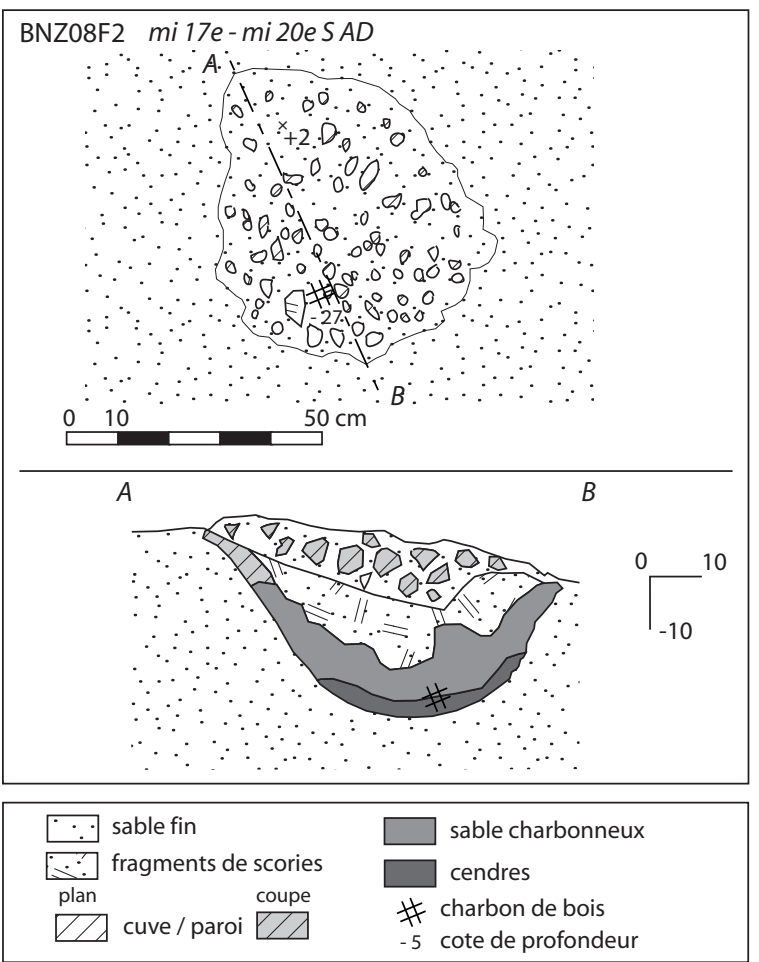

Figure 5. Plan et coupe du bas fourneau à scorie piégée en fond de four (SPff) BNZ08F2.

\section{Bas fourneau à Scorie Piégée en anneau (SPa)}

Les bas fourneaux BKW08F1, KG08F2, KG08F1 (Bassin versant de Saga Gorou) et GBi09F1 (Goroubi) (Fig. 6) ont produit des scories en anneau. La cuve est généralement mal conservée, mais sa forme peut être déduite de la morphologie de la scorie. Elle forme une excavation de $80 \mathrm{~cm}$ de diamètre environ et est constituée d'un fond plat et d'une paroi verticale. Aucun enduit ne semble être appliqué sur les parois, seule une pellicule argilo-sableuse indurée est parfois repérée sur la scorie, elle est liée à la chauffe des particules argilo-sableuses du sol au contact du fluide en fusion. Le centre de la cuve est occupé par un îlot sablo-argileux qui donne à la cuve une forme en anneau. Cet îlot central présente généralement différents gradients de chauffe (KG08F2). La scorie, le plus souvent poreuse et contenant de nombreuses empreintes d'éléments périssables (paille, charbons de bois) forme un anneau dont l'épaisseur est comprise entre 8 et $15 \mathrm{~cm}$ et la hauteur atteint au plus $40 \mathrm{~cm}$. Certaines scories, dont le volume est plus abondant, ont recouvert l'amas central, elles constituent ainsi un bloc creux dont la forme s'apparente à une cloche (KG08F2). À l'inverse, le volume de scorie peut être en quantité trop faible pour remplir entièrement l'anneau de la cuve (BKW08F1).

\section{Bas fourneaux à Scorie Piégée en cylindre (SPc)}

À Garbel dans le bas versant de Saga Gorou, les bas fourneaux GB08F8 et GB08F17 ont produit des scories en forme de petit cylindre vertical de $15 \mathrm{~cm}$ de diamètre et, au maximum, de $25 \mathrm{~cm}$ de haut (Fig. 7). Aucune structure n'est retrouvée, seule la scorie subsiste. La scorie est compacte, son extrémité inférieure est arrondie et la partie supérieure est irrégulière. Elle correspond manifestement à l'emplacement de l'éponge de fer qui a été récupérée par les métallurgistes. Entre la scorie et le substrat sableux une fine couche de matériau sabloargileux très induré est présente ; elle est probablement liée à la chaleur qu'engendre la scorie en fusion, qui modifie les propriétés du substrat sableux en contact direct avec elle (GB08F8). Tous les bas fourneaux de ce type sont tronqués juste au-dessus de la scorie, étant donné sa morphologie, nous pouvons supposer que la partie supérieure de la cuve a été plus large afin de faciliter la mise en place des tuyères et de la cheminée. Le fond de la cuve forme ainsi un surcreusement dans lequel est piégée la scorie.

\section{Bas fourneaux à scorie coulée (SC)}

Ce type de bas fourneau est caractérisé par l'évacuation partielle de la scorie à l'extérieur de la structure par un ou plusieurs orifice(s) placé(s) au fond de la cuve et non par une porte, ces bas fourneaux se déclinent en quatre variantes :

\section{Écoulement vertical (SC-v)}

Un mode d'évacuation original de la scorie a été mis en évidence sur les bas fourneaux issus des sondages GB08F1, GB08F2, GB08F6, LG09F1 et BFD08F4 (Figs. 8, 9 et 10). Les scories coulées sont situées sous la cuve, soit en contact avec elle, soit près de $60 \mathrm{~cm}$ en-dessous, elles sont principalement présentes sous forme de blocs massifs surmontés d'une protubérance. La forme la plus simple et commune à tous ces blocs est celle d'un cylindre en position verticale dont les dimensions atteignent 40 à $50 \mathrm{~cm}$ de haut et près de $50 \mathrm{~cm}$ de diamètre, ce qui équivaut à un volume d'environ $0,1 \mathrm{~m}^{3}$. La forme peut être très irrégulière, elle dépend de la morphologie de la fosse dans laquelle les scories se sont figées. Celles-ci montrent généralement des niveaux stratifiés qui correspondent à une succession de coulures en forme de cordons accolés les uns aux autres. Les coulures se répandent à partir d'un point source qui forme généralement en fin de réduction une proéminence sur la partie sommitale du bloc. Les bords du cylindre ont une surface rugueuse qui témoigne d'un contact avec le substrat sableux. 

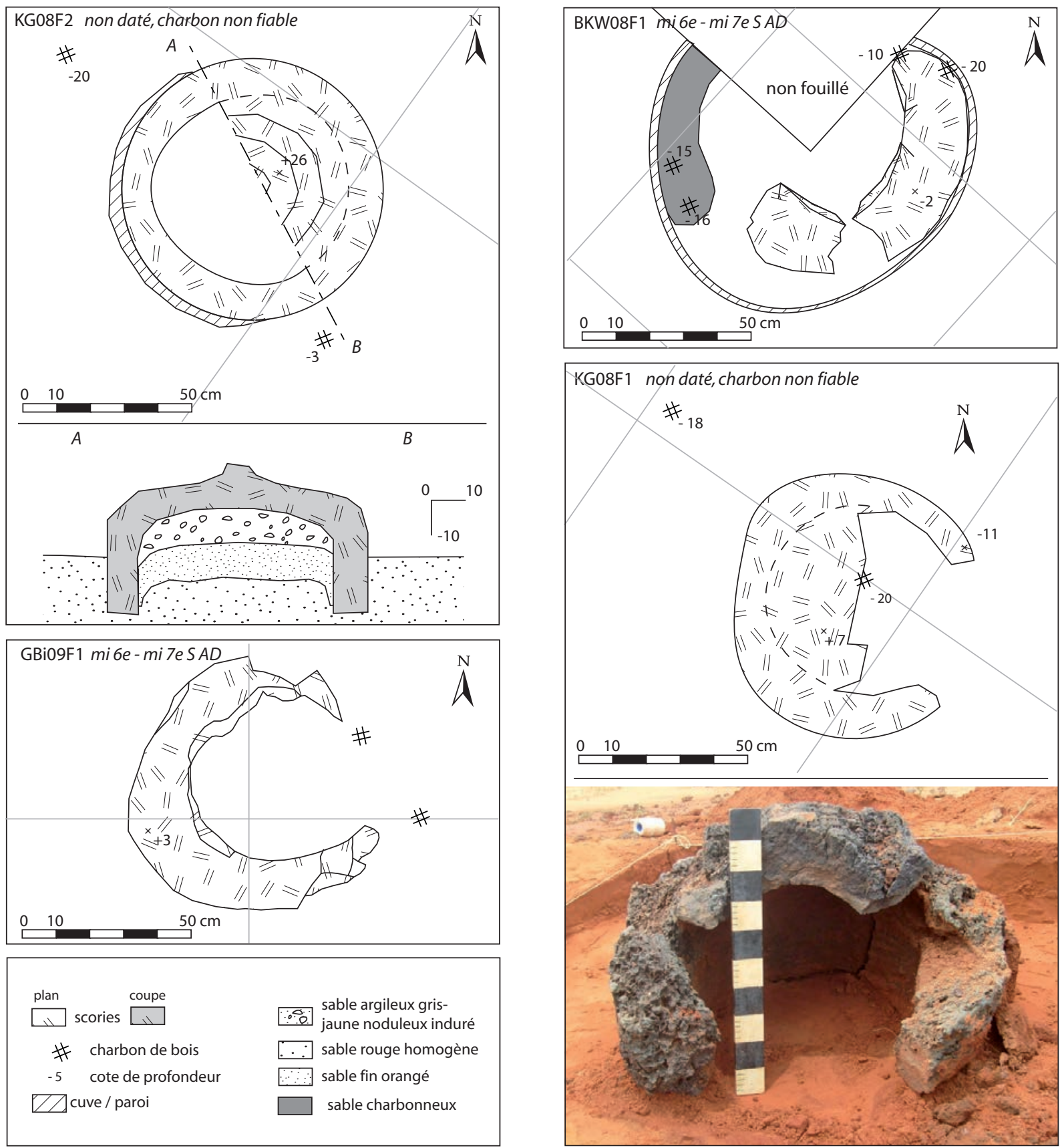

Figure 6. Plan et coupe des bas fourneaux à scorie piégée en anneau (SPa) BKW08F1, KG08F1, KG08F2 et GBi09F1.

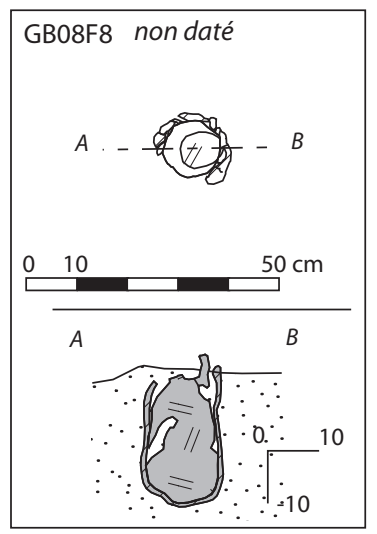

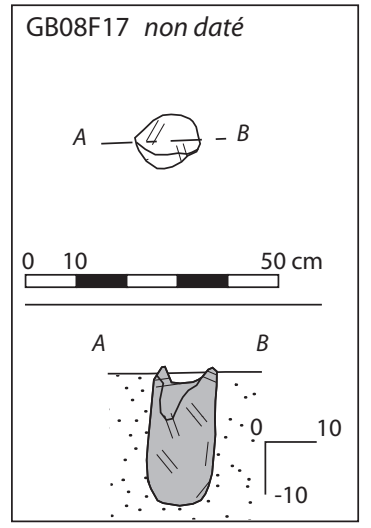

\begin{tabular}{ll}
\hline plan & coupe \\
\hline$\square$ cuve / paroi & scories \\
sable fin &
\end{tabular}

Figure 7. Plan et coupe des bas fourneaux à scorie piégée en cylindre (SPc) GB08F8 et GB08F17. 


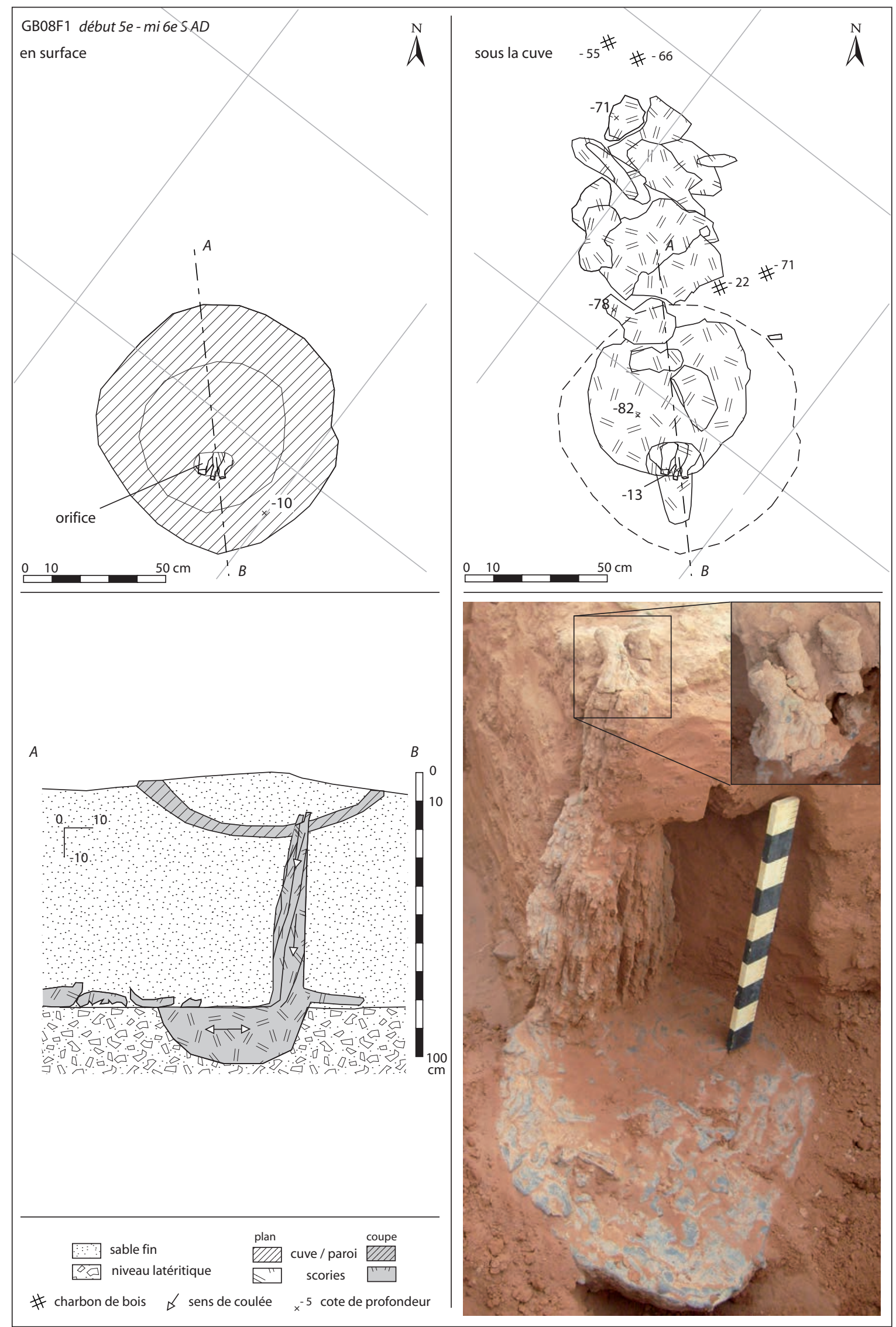

Figure 8. Plan et coupe de bas fourneaux à scorie coulée à écoulement vertical (SC-v) GB08F1. Photographie de la scorie en place et détail sur trois amorces de coulures verticales qui traversent la cuve et forment une colonne pour rejoindre le bloc massif dans la fosse sous-jacente. 

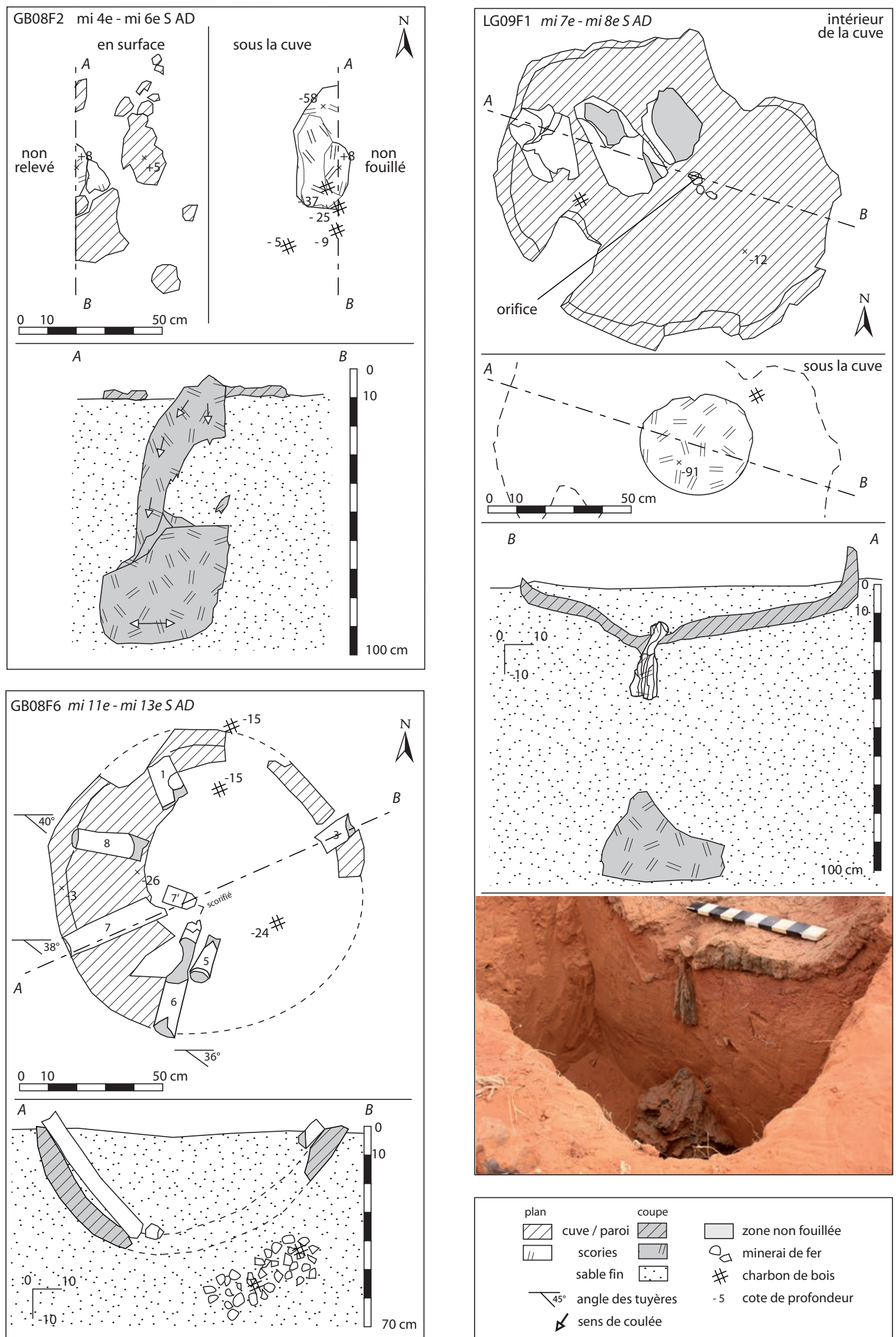

Figure 9. Plan et coupe de bas fourneaux à scorie coulée à écoulement vertical (SC-v) GB08F2, LG09F1 et GB08F6. Photographie de LG09F1 en cours de fouille montrant la coulure de la scorie à travers la cuve au dessus d'un bloc massif de scorie. 
À Bilfouda dans le bassin versant de Saga Gorou, les bas fourneaux GB08F1 et GB08F2 (Figs. 8 et 9 ) ont permis d'observer une longue coulure verticale semblable à celle de la cire d'une bougie ; elle est formée de plusieurs petites coulures accolées les unes aux autres ou d'une coulée unique. Ces coulures verticales sont issues d'orifices de 2 à $5 \mathrm{~cm}$ de diamètre percés au fond de la cuve. Leur longueur peut atteindre $60 \mathrm{~cm}$ et former une colonne entre le haut du bloc massif de scorie et la cuve. Cette disposition montre que la scorie qui se forme dans la cuve est évacuée par gravité dans une fosse sous-jacente, dans laquelle elle s'écoule par goutte à goutte ou en continu. Quand la colonne est absente, des coulures verticales sont visibles sous la cuve à l'aplomb de la proéminence située sur la partie sommitale du bloc massif de scorie. L'extrémité de la coulure est soit conservée et se termine comme une goutte figée avant sa chute, soit elle est sectionnée. $\mathrm{La}$ proéminence située à l'aplomb des orifices correspond manifestement au point d'impact de la scorie liquide tombant de la cuve (LG09F1 et BFD08F4) (Figs. 9, 10 et 11 ).

La fouille du bas fourneau GB08F1 (Fig. 8) a permis de collecter en plus des scories coulées sous la cuve des fragments de scories de type piégé. Elles sont peu épaisses (entre 1 et $5 \mathrm{~cm}$ ) et cintrées. La surface convexe est rugueuse et témoigne du contact avec une surface en creux, contrairement à la face concave qui est très irrégulière avec des traces d'arrachement. $\mathrm{La}$ scorie est toujours compacte, les bords des plaques sont abrupts, ce qui témoigne de cassures nettes. Sur certaines faces convexes, des protubérances cylindriques sont observées, leur extrémité est toujours brisée et leur diamètre n'excède jamais $5 \mathrm{~cm}$. Ces scories cintrées sont clairement issues de la cuve dont elles moulent les courbures. Les protubérances que nous avons décrites sur certaines d'entre elles correspondent au moulage des orifices qui traversent la cuve et permettent l'évacuation de la scorie ; elles sont identifiées comme l'amorce de la coulée verticale observée dans les bas fourneaux GB08F1 et GB08F2. Ces fragments correspondent donc à la scorie restée dans la cuve sans avoir pu être évacuée par écoulement à la fin du processus de réduction. Cette scorie de fond de cuve est fracturée et rejetée lors de la récupération de l'éponge de fer. Ces fragments de scorie ont été retrouvés au même niveau altimétrique que le fond de la fosse, soit près de $80 \mathrm{~cm}$ sous le niveau du sol (Fig. 8). Cette configuration évoque la présence d'une tranchée, située devant le bas fourneau, qui est restée ouverte pendant tout le processus de réduction puisque, au moment de la récupération de l'éponge de fer, les fragments de la scorie de fond de cuve ont pu chuter au fond de cette tranchée. La fonction de cette tranchée est manifestement de permettre le creusement de la fosse située sous la cuve pour recevoir la scorie. Ceci suggère que la fosse était creusée après la confection de la cuve.
Le système de ventilation est assuré par des tuyères qui sont très rarement préservées, excepté pour le bas fourneau GB08F6 (Fig. 9) pour lequel la réduction a échoué. En effet, de nombreux blocs de minerai et quelques charbons de bois se retrouvent sous la cuve dont le fond est éventré. La préservation de la plupart de ces tuyères serait ainsi liée à l'effondrement de la cuve avec le minerai et le charbon de bois dans la fosse sous-jacente peu de temps après le début de la réduction. La faible quantité de charbon de bois dans ces niveaux suggère qu'il ait été récupéré. Les plus longues tuyères conservées mesurent entre 50 et $65 \mathrm{~cm}$. Six sont préservées, mais leur disposition permet de penser que leur nombre était de huit, elles sont espacées régulièrement sur tout le pourtour de la paroi de la cuve avec un pendage compris entre 35 et $40^{\circ}$. Les tuyères 7 et 8 montrent que l'extrémité qui converge vers le centre de la cuve est plus étroite et scorifiée.

Ce type de bas fourneaux est le plus représenté. Sa particularité est de ne pas disposer d'une large ouverture pour évacuer la scorie à l'extérieur de la cuve. En effet, seul un ou plusieurs orifices au fond de la cuve sont utilisés pour séparer par écoulement dans une fosse la scorie de la loupe de fer qui reste dans la cuve. À l'extérieur de celle-ci, la scorie se fige sous la forme de blocs massifs qui créent la contre empreinte de la fosse. Quand le sol est érodé, ces blocs sont visibles directement, mais s'il est préservé, les bas fourneaux sont identifiables uniquement par la présence d'une cuve. Celle-ci contient généralement des fragments de scorie piégée enforme de plaques cintrées issues du moulage du fond de la cuve. Celui-ci est percé d'orifices par lesquels la scorie s'écoule lors de la réduction et constitue des coulures verticales quand elle se fige.

\section{Écoulement latéral (SC-1)}

Dans cette variante, les scories ne sont pas évacuées verticalement, mais latéralement à la cuve. Étant donné sa fragilité, la coulée est rapidement démantelée si elle n'est pas recouverte par le sable. Elles ne sont donc conservées que dans les zones non érodées. La forme des scories est variée, elle dépend de la configuration de la surface sur laquelle la scorie s'écoule. Tous ces bas fourneaux sont situés dans le bassin versant de Saga Gorou. Le sondage GB08F5 (Fig. 12) a révélé trois bas fourneaux, deux avec une fine coulée de scorie libre de près d'un mètre de long et l'autre avec une coulée à laquelle est accolé un bloc à son extrémité. Aucune cuve n'a été mise au jour, elles ont toutes été arasées par l'érosion. Pour BFD09F5 (Fig. 12), la coulée est plus épaisse et repose sur un niveau latéritique très induré sous la surface du sol. Les bords droits de la coulée suggèrent un effet de paroi induit par la présence d'une tranchée. Le point source de la coulée est conservé, il est matérialisé par une protubérance semblable à un petit cylindre qui parait communiquer avec la cuve partiellement conservée. Le 


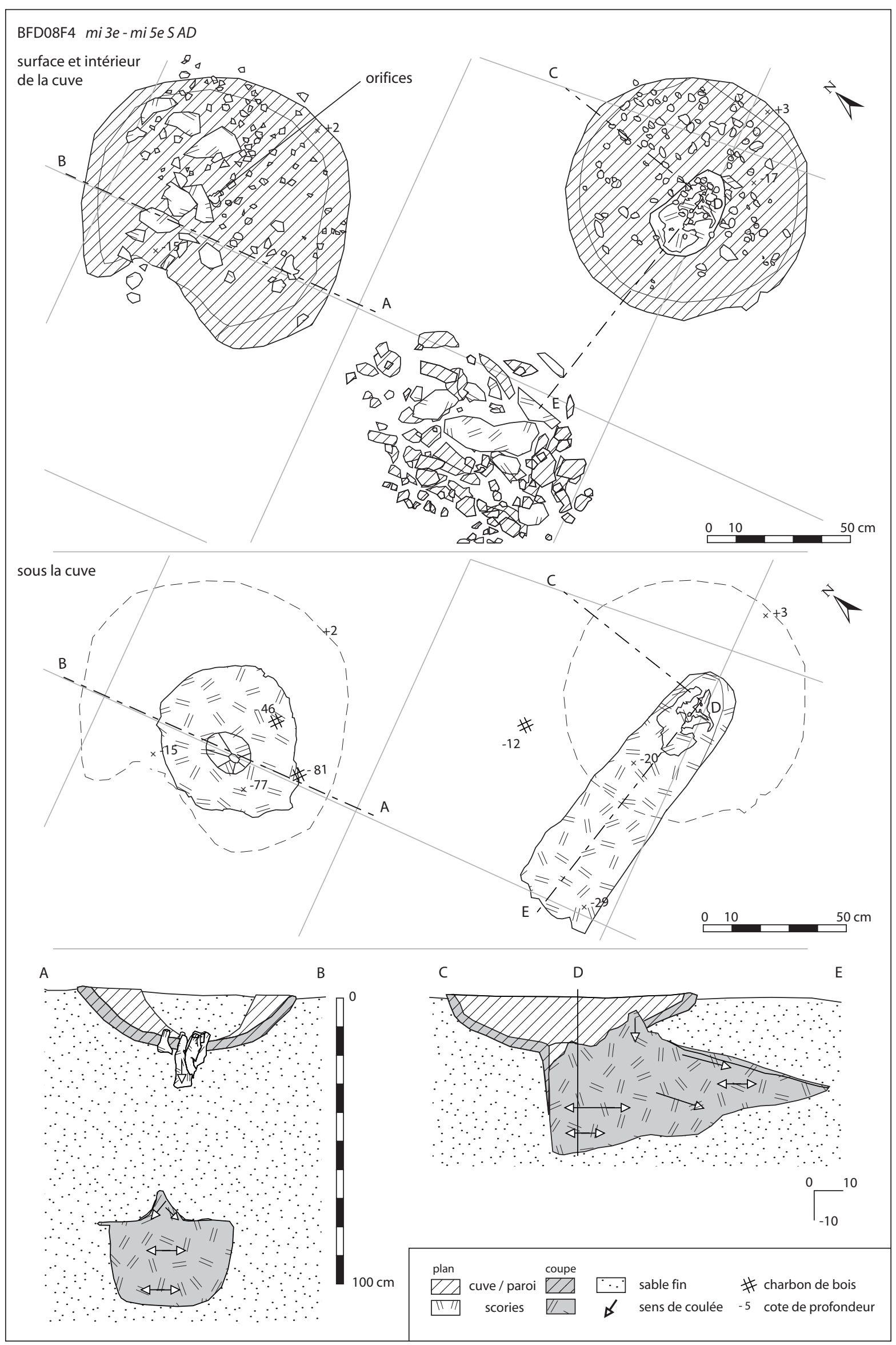

Figure 10. Plan et coupe du bas fourneau à scorie coulée à écoulement vertical (SC-v) BFD08F4. 

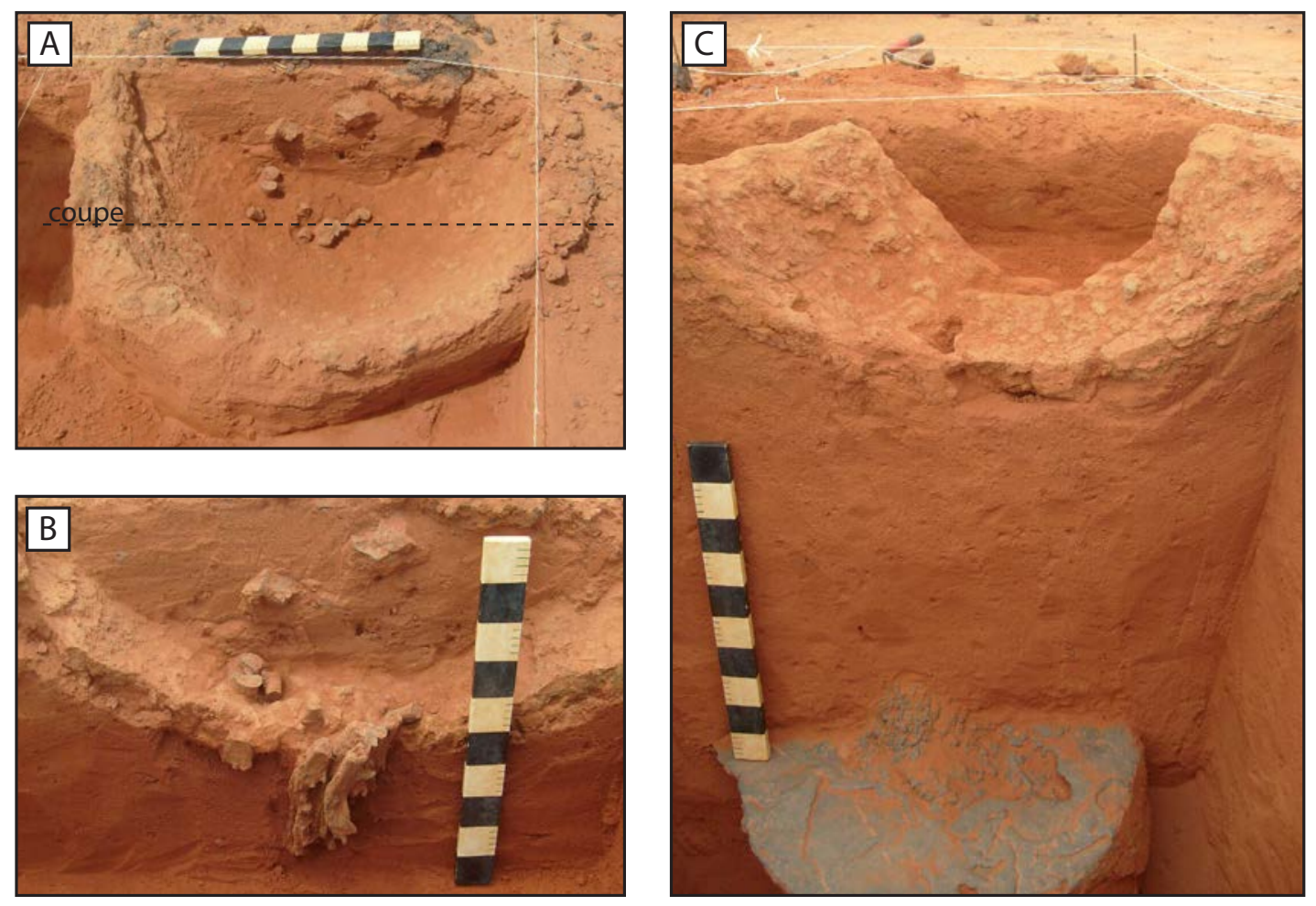

Figure 11. Photographies du bas fourneau nord BFD08F4 de type scorie coulée à écoulement vertical (SC-v). A : cuve sablo-argileuse indurée et amorces de coulures verticales au fond de la cuve ; B : coupe de la cuve. Les coulures traversent la cuve et s'interrompent ; $\mathrm{C}$ : à l'aplomb des coulures (déjà prélevées lors de la prise de vue) présence d'un bloc massif dans une fosse à près d'un mètre de profondeur.

bas fourneau GB08F9 (Fig. 13) a également une coulée épaisse, elle est positionnée sous le niveau de la cuve et débute verticalement puis devient horizontale. La cuve de $80 \mathrm{~cm}$ de diamètre est bien conservée. De forme hémisphérique, l'excavation creusée dans le sable a été enduite d'une couche d'argile sableuse dont l'épaisseur est comprise entre 5 et $10 \mathrm{~cm}$. Le fond de la cuve est traversé de part en part par l'amorce d'une coulée de scorie qui, bien que rompue, peut être connectée au point source de la coulée situé sous la cuve. L'évacuation de la scorie s'effectue ainsi par un orifice aménagé dans la paroi de la cuve. La scorie est ensuite recueillie dans une tranchée creusée au devant de la cuve. Deux tuyères ont été identifiées, l'une d'entre elles est préservée en place et positionnée de manière oblique avec un angle de $45^{\circ}$ sur le bord de la cuve.

\section{Écoulement hybride}

Les bas fourneaux SG08F2 à Saga Gorou et BNZ08F1 à Banizoumbou (Fig. 14) ont laissé des formes hybrides combinant un bloc massif à pointe et une coulée. Elle est constituée à partir de deux sens d'écoulement qui prennent une direction opposée : l'une forme un bloc plus ou moins épais et d'un diamètre compris entre 40 et $50 \mathrm{~cm}$ et l'autre forme une coulée le plus souvent assez courte et peu épaisse. Ces coulures sont issues d'une même source située entre le bloc et la coulée.
Elles suggèrent que la fosse située sous la cuve pouvait prendre différentes formes, tout en ayant le même principe de fonctionnement.

Le peu d'exemplaires étudiés et la mauvaise conservation des structures ne nous a pas permis d'en saisir le fonctionnement. La présence de deux coulées, l'une principale dans une fosse et l'autre de plus faible volume dans un chenal reste difficile à expliquer.

Écoulement de petit volume (SC-pv)

Dans le bassin versant de Saga Gorou, un dernier ensemble est constitué des bas fourneaux GB08F10, GB08F12 et SG09F4 (Figs. 15 et 16). Ils sont caractérisés par des blocs massifs de petites dimensions dont le volume est jusqu'à dix fois inférieur à ceux décrits précédemment, il n'excède pas $0,02 \mathrm{~m}^{3}$. La forme des scories est très variable, ce qui est probablement lié à une faible production de scories. Cela laisse plus de liberté pour le choix de la fosse réceptacle. Certaines sont des blocs cylindriques, d'autres s'apparentent à une coulée sur rampe. La protubérance qui démontre qu'il s'agit bien de scories coulées est généralement placée sur la partie centrale du bloc, mais peut également être latérale. Pour cause de forte érosion des sols, aucune structure argilo-sableuse (cuve, tuyères, cheminée) n'a été observée à la fouille. 

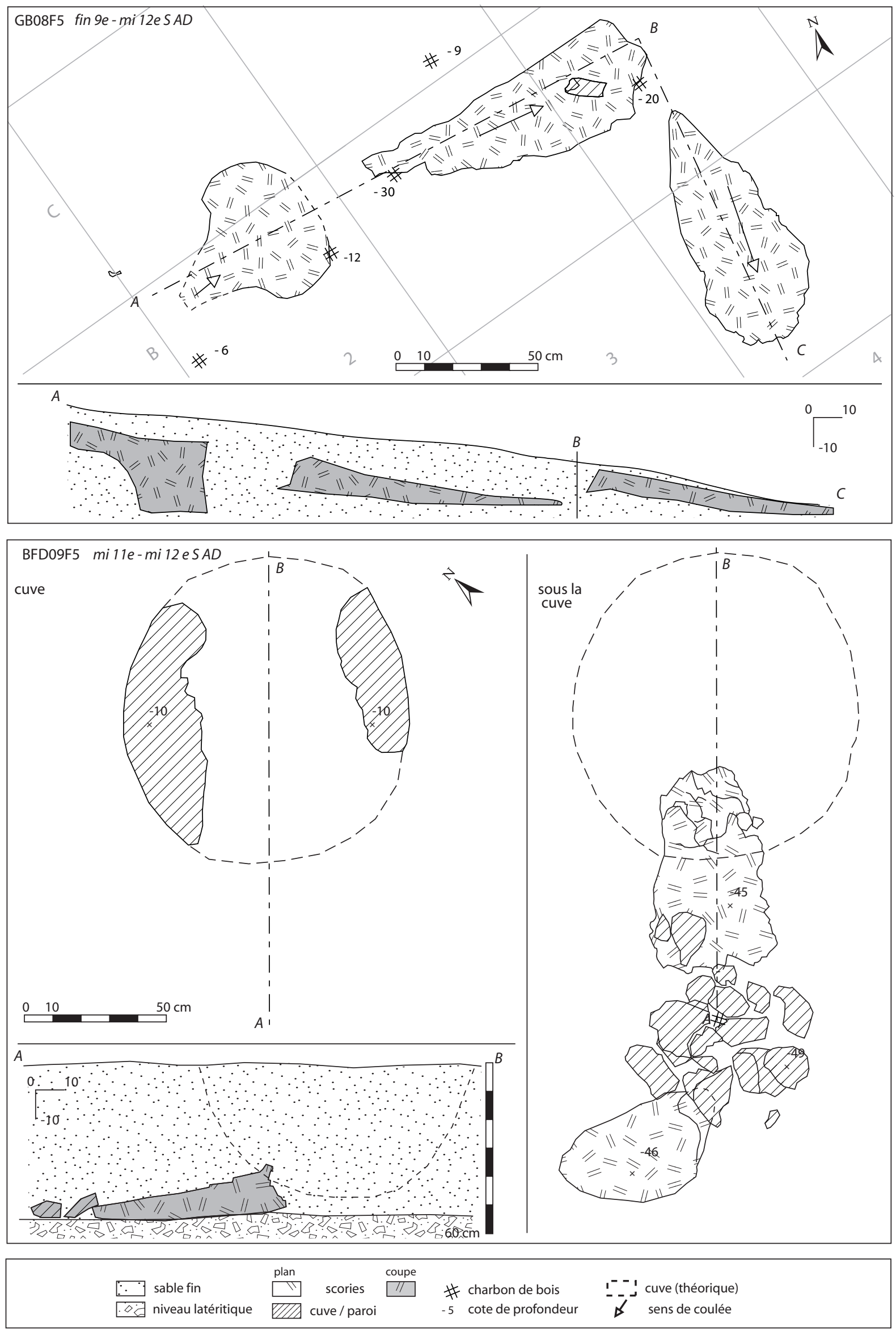

Figure 12. Plan et coupe de bas fourneaux à scorie coulée à écoulement latéral (SC-1) GB08F5 et BFD09F5. 

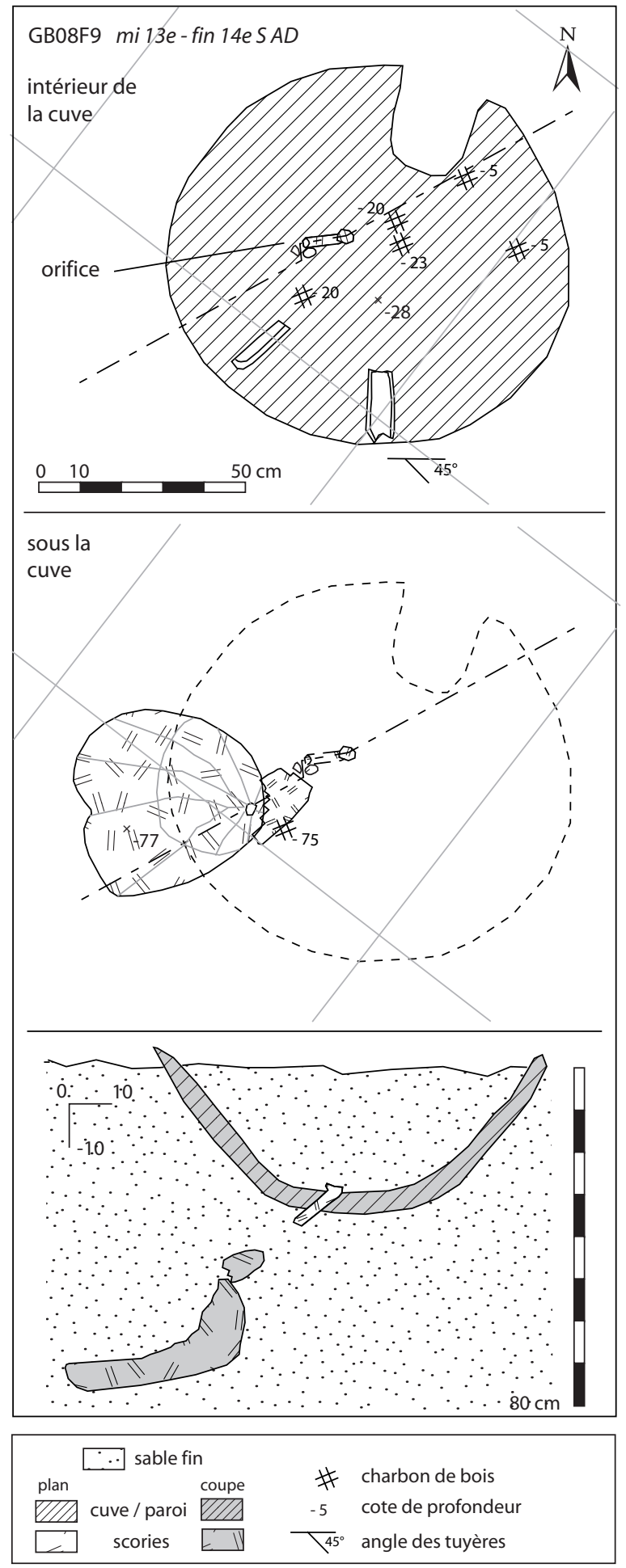

Figure 13. Plan et coupe du bas fourneau à scorie coulée à écoulement latéral (SC-1) GB08F9.
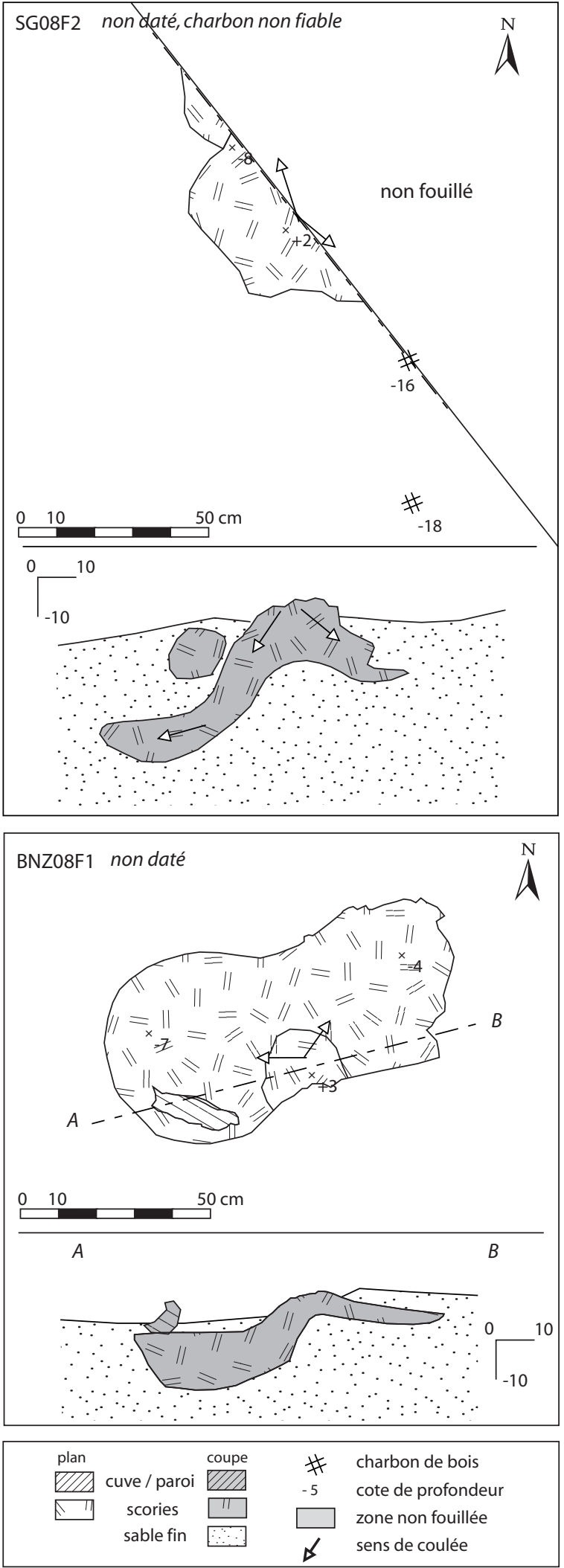

Figure 14. Plan et coupe de bas fourneaux à scorie coulée à écoulement hybride (SC-hyb) SG08F2 et BNZ08F1. 


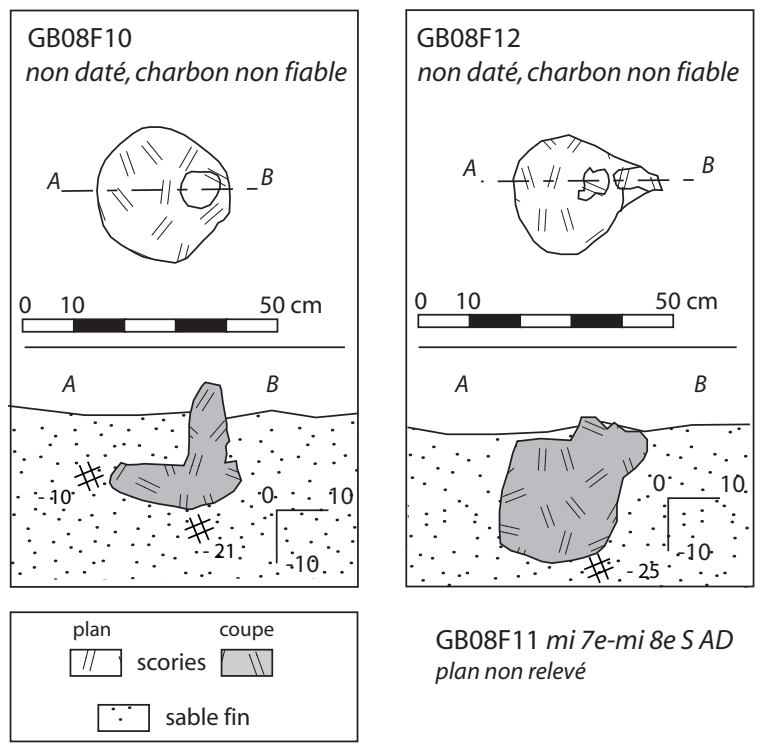

Figure 15. Plan et coupe de bas fourneaux à scorie coulée de petit volume (SC-pv) GB08F10 et GB08F12.
Ces petits blocs massifs sont issus de bas fourneaux à scorie coulée de petites dimensions. La forme de la scorie est très aléatoire, elle est évacuée de la cuve soit verticalement soit latéralement.

\section{Chronologie}

Les données chronologiques se concentrent dans la région de Saga Gorou où 23 dates ont été validées. Huit autres sont réparties à Banizoumbou (2), Louguel (3), Gobéri (2) et dans la vallée du Goroubi (1) (Fig. 17).

Les données spatiales, typologiques et chronologiques des 35 bas fourneaux étudiés sont regroupées sur une carte de synthèse (Fig. 18). Pour le secteur de Saga Gorou qui est le mieux doté, les bas fourneaux datés représentent moins de $1 \%$ des bas fourneaux recensés, il est donc délicat d'établir des conclusions typo-chronologiques précises. De même, la répartition spatiale de ces différents types et leur regroupement restent

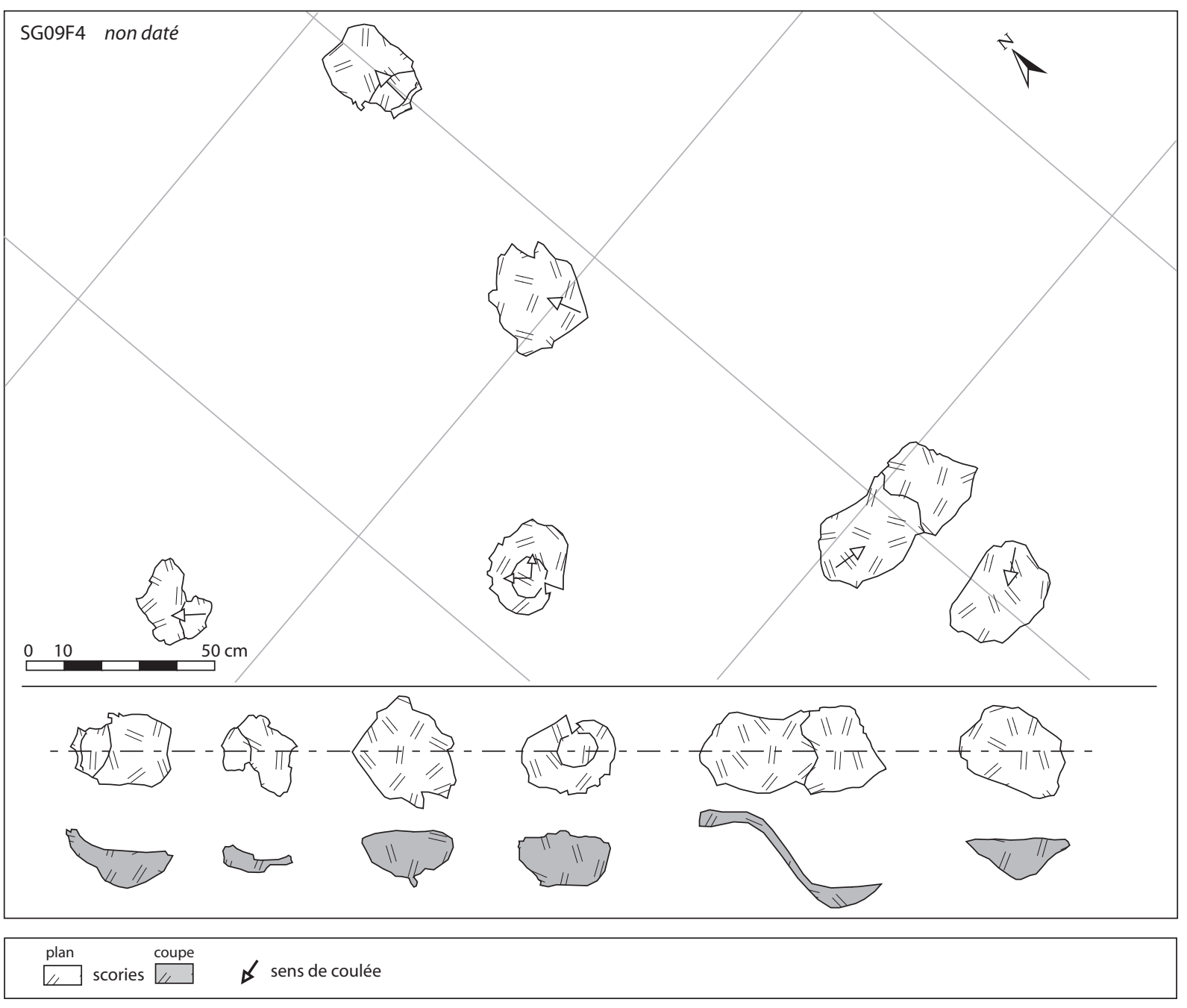

Figure 16. Plan et coupe du bas fourneau à scorie coulée de petit volume (SC-pv) SG09F4. 


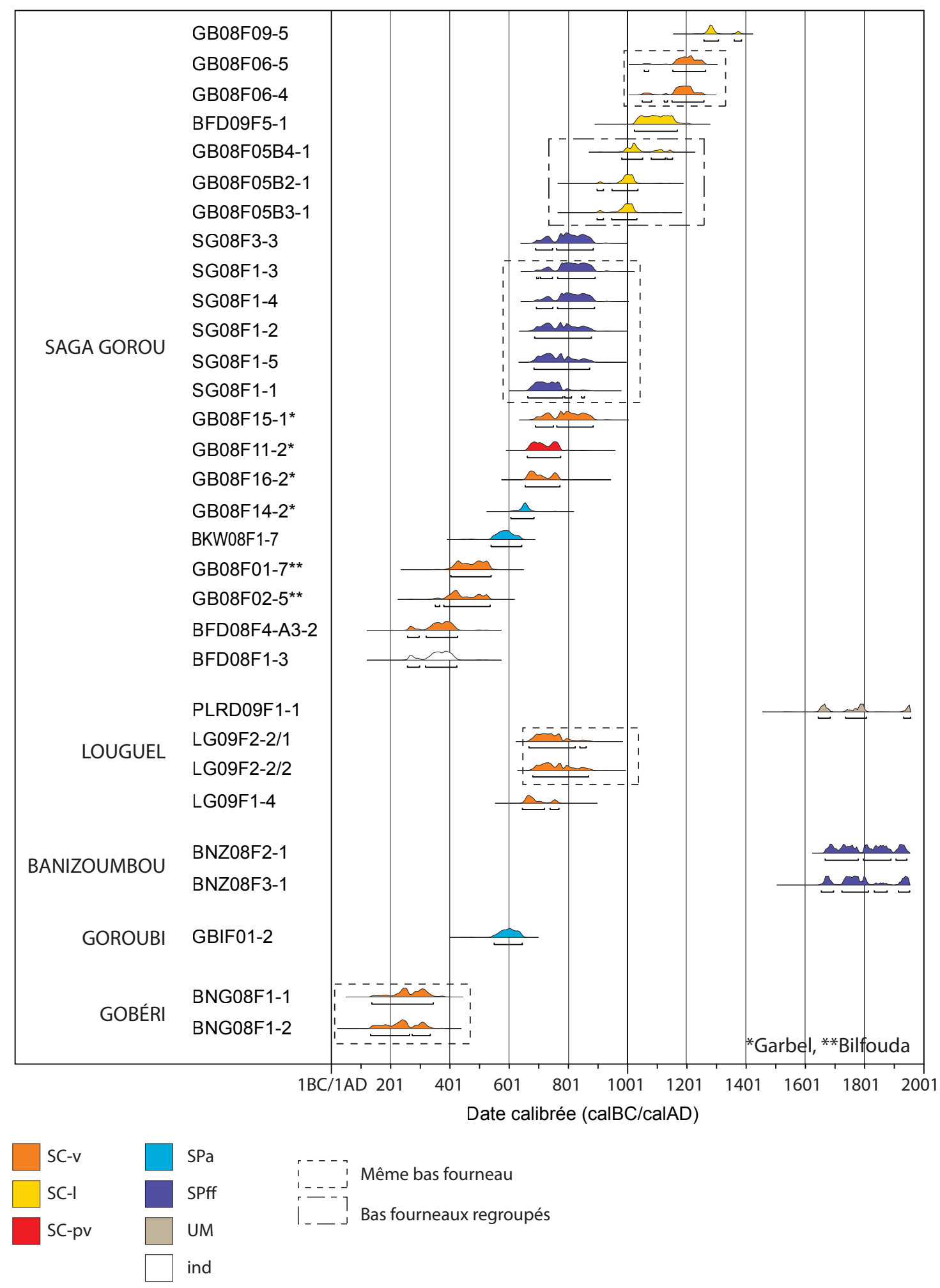

Figure 17. Résultat des 39 datations radiocarbone issues de bas fourneaux de réduction du fer. Calibration Oxcal 4.1. (BRONK RAMSEY 2009).

difficiles à interpréter et à extrapoler. L'information chronologique est directement liée à la préservation du charbon de bois qui dépend elle-même du type de bas fourneaux et de l'érosion des sols sur lesquels ils sont construits. En tenant compte de ces limites taphonomiques, nous présentons succinctement le bilan de ces données chronologiques. 

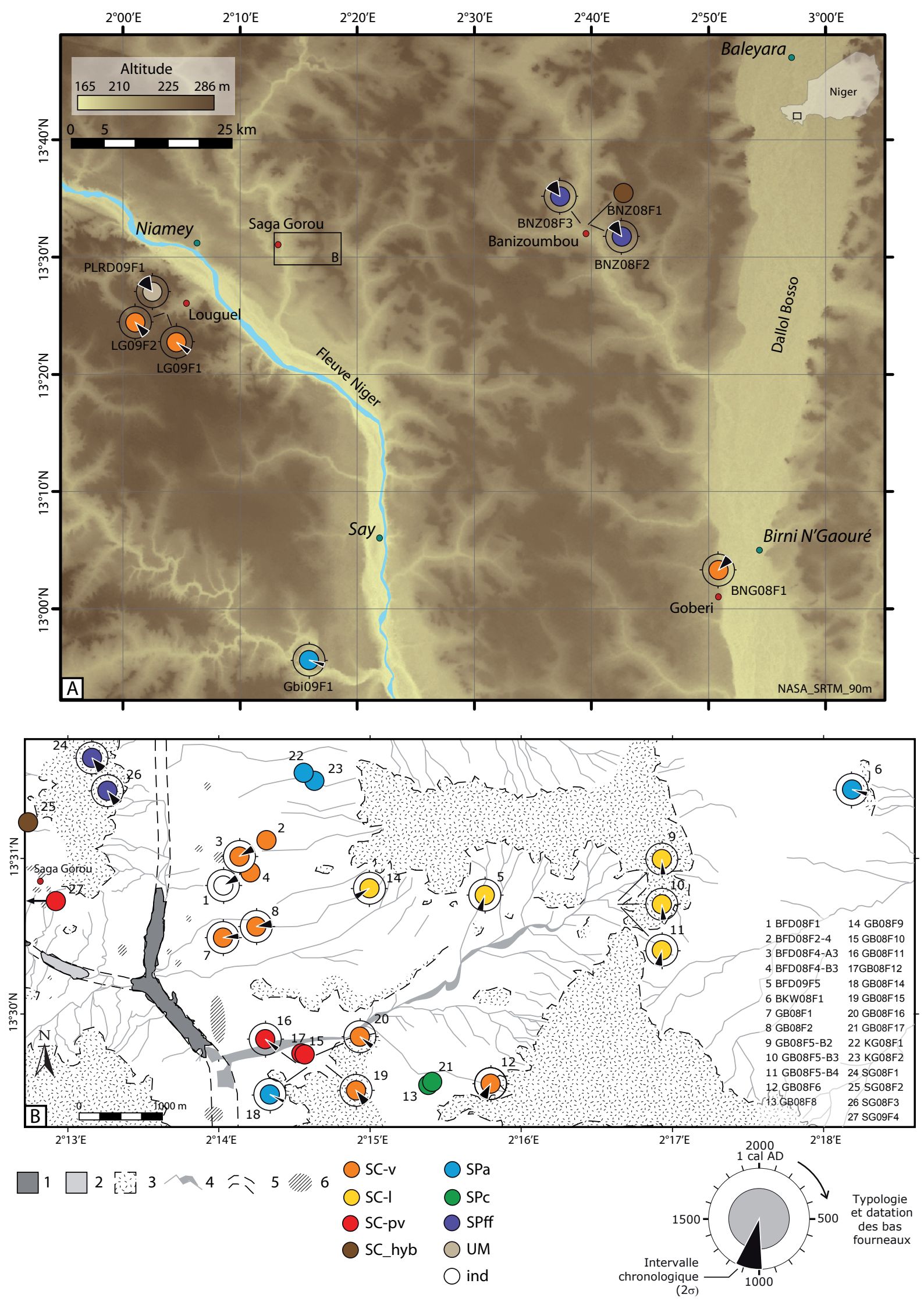

Figure 18. Carte de répartition des bas fourneaux datés et représentation graphique des datations. A : Le Sud-ouest du Niger, de la vallée du fleuve au Dallol Bosso ; B : Bassins versants de Saga Gorou ; 1 : Lac permanent ; 2 : Lac temporaire ; 3 : Plateaux et haut et moyen glacis ; 4 : Ravines ; 5 : Réseau fluvial ancien ; 6 : Habitats actuels. 


\section{Bassin versant de Saga Gorou}

La métallurgie du fer se développe dans les environs de Saga Gorou à partir du $4^{\mathrm{e}}$ siècle de notre ère ; les bas fourneaux qui ont bénéficié du plus grand nombre de datations sont ceux à scorie coulée à écoulement vertical (SC-v), car ils sont très fréquents et leur architecture favorise la préservation des charbons de bois dans les zones non érodées. Selon la datation de six bas fourneaux, leur fonctionnement s'étendrait du $4^{\mathrm{e}}$ au début du $13^{\mathrm{e}}$ siècles, toutefois deux périodes ne sont pas couvertes, vers les $6^{\mathrm{e}}$ et $7^{\mathrm{e}}$ siècles et vers les $9^{\mathrm{e}}$ et $10^{\mathrm{e}}$ siècles. S'ensuivent les bas fourneaux à scorie piégée en anneau ( $\mathrm{SPa}$ ) pour lesquels deux dates fiables ont été obtenues, elles indiquent une utilisation vers le $6^{\mathrm{e}}$ et le $7^{\mathrm{e}}$ siècles. Au $7^{\mathrm{e}}$ siècle apparaissent également les bas fourneaux à scorie piégée en fond de four (SPff), pour lesquels nous disposons de six dates pour deux bas fourneaux. Ils sont construits principalement sur les plateaux au nord de Saga Gorou, ce type ne semble plus être utilisé au-delà du $9^{\mathrm{e}}$ siècle. Les bas fourneaux à scorie coulée de petit volume (SC-pv) sont peu pro- pices à la préservation de charbons de bois, un seul a pu être daté, il est utilisé entre le milieu du $7^{\text {e }}$ et la fin $\mathrm{du} 8^{\mathrm{e}}$ siècles. Les bas fourneaux à scorie coulée latérale (SC-1) ont été datés par cinq fois, trois d'entre eux sont distants de moins d'un mètre, ils ont tous fonctionné entre $1 \mathrm{e} 10^{\mathrm{e}}$ et $1 \mathrm{e} 14^{\mathrm{e}}$ siècles. Pour la deuxième moitié du deuxième millénaire de notre ère, la région de Saga Gorou ne dispose d'aucune date fiable.

Il faut noter que les bas fourneaux à scorie piégée en cylindre (SPc) n'ont pu être datés, le mode de fonctionnement et l'architecture réduisent fortement les possibilités de découvrir des charbons de bois qui y seraient associés. Toutefois, les vestiges de ces bas fourneaux se rapprochent des descriptions ethnographiques établies par N. ÉCHARD (1983) dans l'Ader et de la reconstitution réalisée à Banizoumbou en 1998 (Fig. 19). Ce type de bas fourneaux à usage unique semble être le seul connu dans le Sud-ouest du Niger par les métallurgistes de la première moitié du $20^{\text {e }}$ siècle; aucun indice ne permet de confirmer qu'ils aient fonctionné antérieurement au $19^{\text {e }}$ siècle.
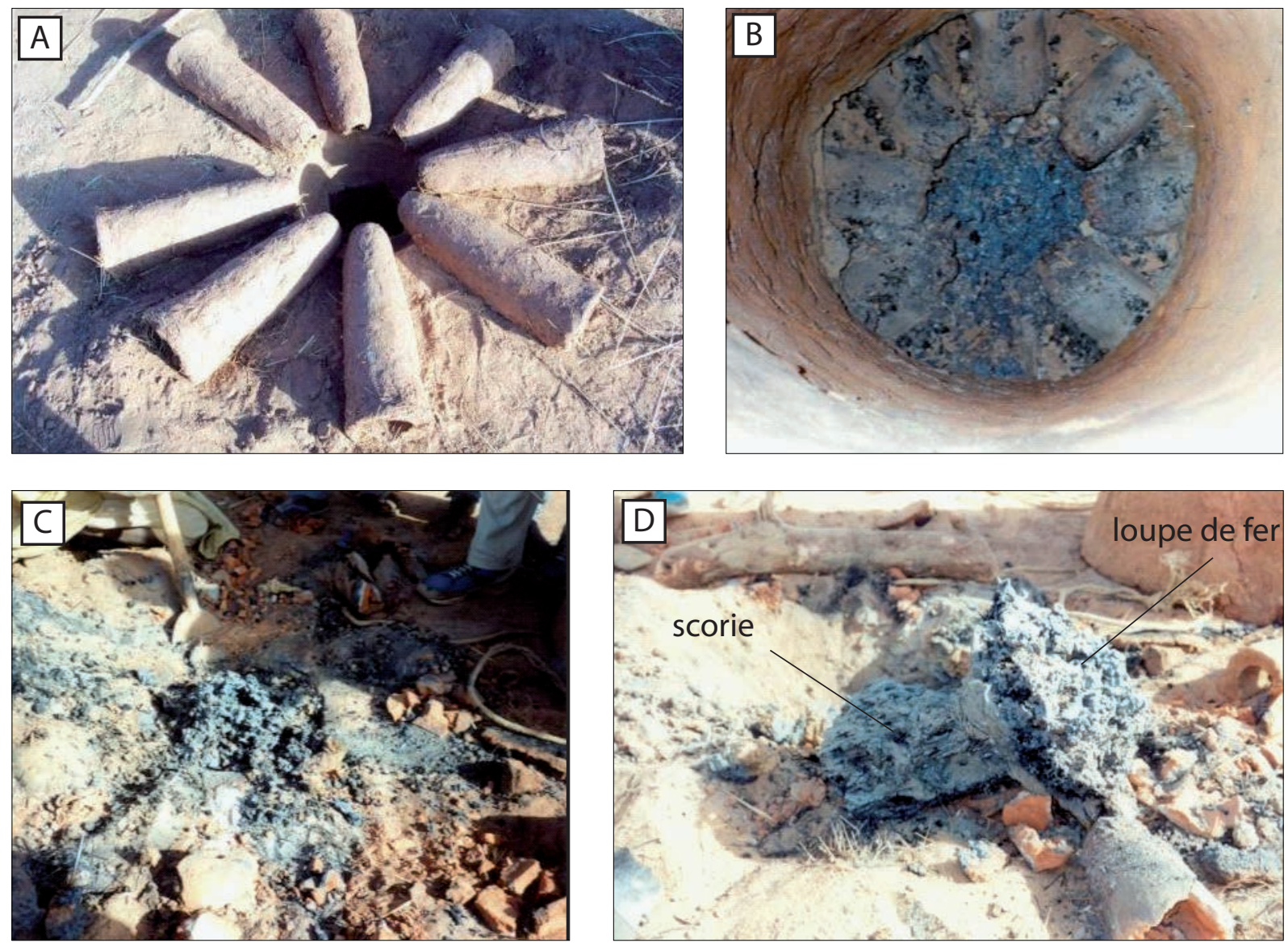

Figure 19. Reconstitution d'un bas fourneau à Banizoumbou en 1998 identique au modèle décrit par N. ÉcHARD (1983) (photo J.L. Rajot). A : Vue sur huit tuyères au tour de la cuve avant la pose de la cheminée. La cuve est cylindrique, le haut est élargi ; $\mathrm{B}$ : Vue à l'intérieur de la cheminée après réduction. Les tuyères et la loupe de fer sont visibles; $\mathrm{C}$ : Dégagement de la loupe de fer après démantèlement de la cheminée et des tuyères ; D. Loupe de fer et scorie encore liées extraites de la cuve. 
Ainsi, l'activité métallurgique dans la région de Saga Gorou s'étend du $4^{\mathrm{e}}$ au $14^{\mathrm{e}}$ siècles période durant laquelle la plupart des types de bas fourneaux sont utilisés. Les enquêtes auprès des populations locales indiquent que la réduction du fer était pratiquée entre le $19^{\mathrm{e}}$ et la première moitié du $20^{\mathrm{e}}$ siècle. Le bas fourneau à scorie piégée en cylindre $(\mathrm{SPc})$ pourrait correspondre à cette période d'activité. L'activité de réduction du fer paraît ainsi s'interrompre durant au moins quatre siècles (du $15^{\mathrm{e}}$ au $18^{\mathrm{e}}$ siècles) comme le suggère l'absence de structure datée de cette période.

\section{Les autres sites sidérurgiques}

Les dates radiocarbone disponibles pour les quatre autres secteurs sont peu nombreuses, ce qui limite leur interprétation. Parmi les dates fiables, nous constatons que les plus anciennes sont celles obtenues à Gobéri où deux charbons de bois d'un même bas fourneau de type $\mathrm{SC}-\mathrm{v}$ sont datés entre la première moitié du $2^{\mathrm{e}}$ siècle et la première moitié du $4^{\mathrm{e}}$ siècle de notre ère, soit peu de temps avant ceux de Saga Gorou.

Dans la vallée du Goroubi, le bas fourneau de type SPa est daté entre la moitié du $6^{e}$ et la moitié du $7^{\mathrm{e}}$ siècles, ce qui est contemporain de ceux de Saga Gorou, distant de $65 \mathrm{~km}$ au nord.

À Louguel, deux bas fourneaux de type SC-v situés au pied du talus de plateau à proximité d'un habitat ancien ont été daté entre le milieu du $7^{\mathrm{e}}$ siècle et la seconde moitié du $8^{e}$ siècle et entre la seconde moitié $\mathrm{du} 7^{\mathrm{e}}$ et la seconde moitié du $9^{\mathrm{e}}$ siècle. Cette période est contemporaine de celle obtenue dans les environs de Saga Gorou.

À Banizoumbou, les deux datations de bas fourneaux à scorie piégée en fond de four (SPff) sont également tardives. Mais à l'inverse de Louguel, les enquêtes menées auprès de la population locale nous précisent que ces bas fourneaux ont été construits au début du $20^{\mathrm{e}}$ siècle. Il faut pourtant noter que la majorité des bas fourneaux observés lors des prospections est principalement de type $\mathrm{SC}$-v, mais les sols très érodés sur lesquels ils sont aujourd'hui situés n'ont pas permis de trouver d'élément de datation.

\section{Discussion}

\section{Des modes de production variés}

Entre le fleuve Niger et le Dallol Bosso, la métallurgie $\mathrm{du}$ fer est pratiquée depuis la première moitié du $2^{\mathrm{e}}$ siècle et plusieurs types de bas fourneaux exclusivement à usage unique ont été construits et ont coexistés, ils témoignent d'un savoir-faire varié qui a perduré au moins jusqu'au $14^{\mathrm{e}}$ siècle (Fig. 20). Leur typologie est basée tout d'abord sur le mode de séparation entre l'éponge de fer et la scorie qui peut être lié à l'appartenance à telle ou telle famille de forgerons. Ensuite, elle repose sur des procédés techniques et architecturaux différents qui résultent soit du savoir-faire des métallurgistes soit des contraintes du milieu. En effet, pour les bas fourneaux à scories piégées les procédés techniques sont nettement différents les uns des autres alors que les bas fourneaux à scorie coulée semblent tous appartenir à un même principe de fonctionnement, où seule la forme de la fosse réceptacle et le volume de scorie diffèrent (Fig. 21).

Les bas fourneaux à scorie piégée en fond de four (SPff) datés entre la fin du $7^{\mathrm{e}}$ et la fin du $9^{\mathrm{e}}$ siècles à Saga Gourou, sont les seuls à être construits sur les plateaux latéritiques car ils ne nécessitent aucune excavation profonde qui aurait été difficile à réaliser dans les niveaux cuirassés très indurés. Ils sont similaires et contemporains de la tradition KRS1 de Korsimoro au Burkina Faso (Serneels et al. 2012). Ceux construit près de mille ans plus tard au $18^{\mathrm{e}}$ siècle à Banizoumbou semblent utiliser un procédé identique, mais ils ne peuvent appartenir à la même tradition métallurgique.

Les bas fourneaux à scorie piégée en anneau (Spa) fonctionnent vers le début du $7^{\mathrm{e}}$ siècle à Saga Gorou et plus au sud sur les rives du Goroubi. Des structures de même type ont également été identifiées par milliers dans la région de Markoye dans la vallée du Béli au nord-est du Burkina Faso où deux dates indiquent un fonctionnement autour de l'an 1000 (FABRE 2009, 2012a, b). Dans cette région, un second modèle de cuve semblable a été mis au jour : au lieu de l'îlot central, une tuyère en position verticale est employée. Ce même agencement est également décrit dans la zone du delta intérieur du Niger au Mali (CHIÈze 1991). À partir de l'étude archéologique, une hypothèse de fonctionnement des bas fourneaux Spa est proposée (Fig. 22).

Les bas fourneaux à scorie piégée en cylindre (SPc) seraient l'un des derniers modes de production du fer utilisés par les métallurgistes avant l'abandon de l'activité au milieu du $20^{\mathrm{e}}$ siècle. Des scories en forme de cylindre et de dimensions similaires ont également été observées et étudiées à Korsimoro au Burkina Faso. Leur production est également tardive, elle est datée dans les environs du $17^{\mathrm{e}}$ siècle (SERneels 2012). Cependant, en l'absence de structures construites dans les deux cas, il est difficile de confirmer qu'il s'agit du même procédé. Pour la région de Saga Gorou, en s'appuyant sur les données ethnographiques de N. Échard et sur la reconstitution réalisée en 1998 (Fig. 19) nous pouvons présenter les différentes étapes qui conduisent à la production de la loupe de fer (Fig. 23). 
A

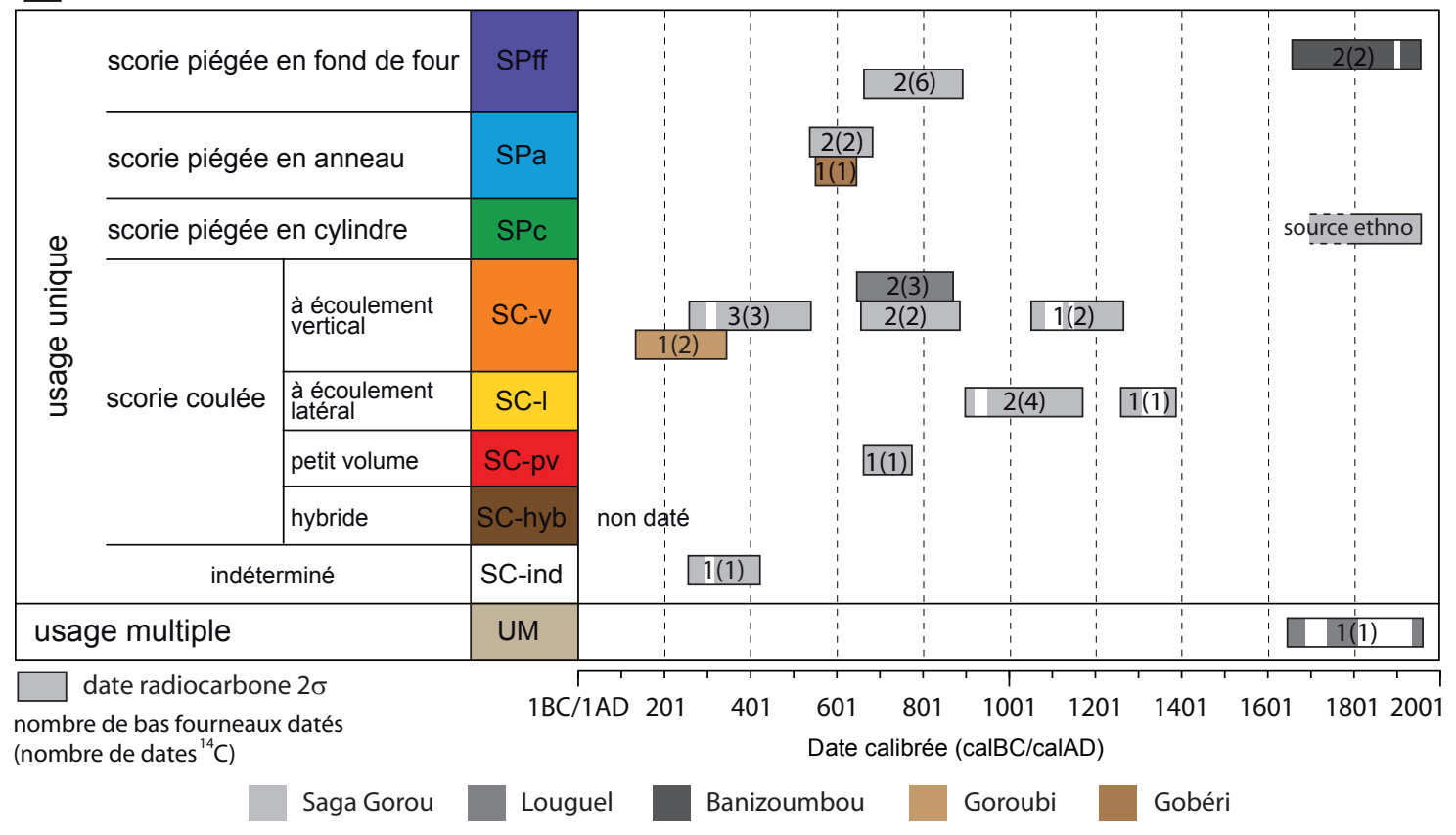

B

\begin{tabular}{|c|c|c|c|c|c|c|c|c|c|c|}
\hline \multirow{3}{*}{ secteur } & \multirow{3}{*}{$\begin{array}{l}\text { usage } \\
\text { multiple }\end{array}$} & \multicolumn{8}{|c|}{ usage unique } & \multirow{3}{*}{\begin{tabular}{|c}
$\mathrm{BF}$ daté \\
$\mathrm{BF}$ fouillé \\
par \\
secteur
\end{tabular}} \\
\hline & & \multicolumn{3}{|c|}{ scorie piégée } & \multicolumn{4}{|c|}{ scorie coulée } & \multirow[b]{2}{*}{$\begin{array}{l}\text { indéter- } \\
\text { miné }\end{array}$} & \\
\hline & & \begin{tabular}{|c|} 
fond \\
de four
\end{tabular} & anneau & cylindre & $\begin{array}{c}\text { écoulement } \\
\text { vertical }\end{array}$ & $\begin{array}{l}\text { écoulement } \\
\text { latéral }\end{array}$ & $\begin{array}{c}\text { petit } \\
\text { volume }\end{array}$ & hybride & & \\
\hline $\begin{array}{c}\text { Saga } \\
\text { Gourou }\end{array}$ & & $\begin{array}{l}\text { SG08F1 } \\
\text { SG08F2 }\end{array}$ & $\begin{array}{c}\text { BKW08F1 } \\
\text { KG08F2 } \\
\text { KG08F1 } \\
\text { GB08F14 }\end{array}$ & $\begin{array}{l}\text { GB08F8 } \\
\text { GB08F17 }\end{array}$ & $\begin{array}{c}\text { GB08F1 } \\
\text { GB08F2 } \\
\text { GB08F6 } \\
\text { BFD08F4-A3 } \\
\text { BFD08F4-B3 } \\
\text { BFD08F2 } \\
\text { GB08F15 } \\
\text { GB08F16 }\end{array}$ & $\begin{array}{c}\text { GB08F5-B2 } \\
\text { GB08F5-B3 } \\
\text { GB08F5-B4 } \\
3 \text { BFD09F5 } \\
3 \text { GB08F9 }\end{array}$ & $\begin{array}{c}\text { GB08F10 } \\
\text { GB08F12 } \\
\text { SG09F4 } \\
\text { GB08F11 }\end{array}$ & SG08F3 & BFD08F1 & $18 / 27$ \\
\hline Louguel | & $\begin{array}{l}\text { PLRD } \\
\text { 09F1 }\end{array}$ & & & & $\begin{array}{l}\text { LG09F1 } \\
\text { LG09F2 }\end{array}$ & & & & & $3 / 3$ \\
\hline $\begin{array}{l}\text { Bani- } \\
\text { :oumbou }\end{array}$ & & $\begin{array}{l}\text { BNZ08F2 } \\
\text { BNZ08F3 }\end{array}$ & & & & & & BNZ08F1 & & $2 / 3$ \\
\hline Goroubi & & & GBi09F1 & & & & & & & $1 / 1$ \\
\hline Gobéri & & & & & BNG08F1 & & & & & $1 / 1$ \\
\hline $\begin{array}{l}\text { BF daté } \\
\text { BF fouille } \\
\text { par type }\end{array}$ & $1 / 1$ & $4 / 4$ & $3 / 5$ & $0 / 2$ & 9/11 & $5 / 5$ & $1 / 2$ & $1 / 4$ & $1 / 1$ & $25 / 35$ \\
\hline
\end{tabular}

Figure 20. Synthèse chrono-typologique. A : Représentation graphique des datations radiocarbone par secteur et par typologie ; B : Tableau de synthèse des bas fourneaux fouillés et datés classés par secteur et par typologie. 


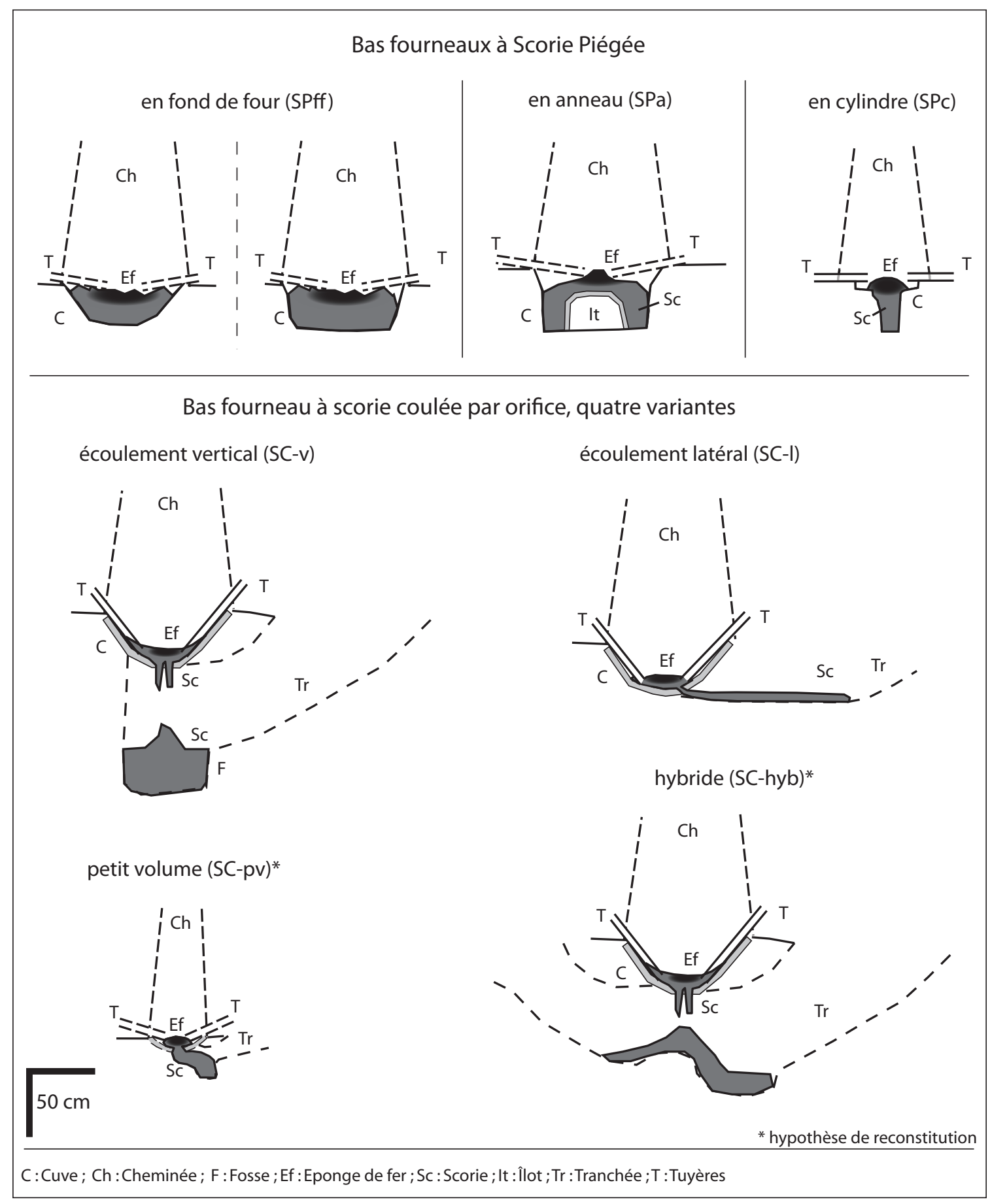

Figure 21. Synthèse typologique des bas fourneaux à usage unique découverts au Sud-ouest du Niger.

Les bas fourneaux à scorie coulée (SC) sont les plus nombreux. La morphologie de la scorie évacuée par un orifice est très variée, elle dépend du contexte local, des conditions de réduction et du facteur humain. Les bas fourneaux à scorie coulée à écoulement vertical semblent être ceux construits par défaut. Les autres modèles seraient produits quand toutes les conditions de mise en œuvre ne sont pas réunies. En effet, la pente du terrain et l'épaisseur du sol sableux influent sur la construction de la fosse réceptacle. Au lieu de creuser sous la cuve une fosse cylindrique, une simple tranchée peut être aménagée latéralement, ce qui produit des coulées latérales. Les paramètres de réduction ont également un rôle prépondérant dans la forme de la scorie coulée, par exemple selon la température de fusion, la scorie peut 


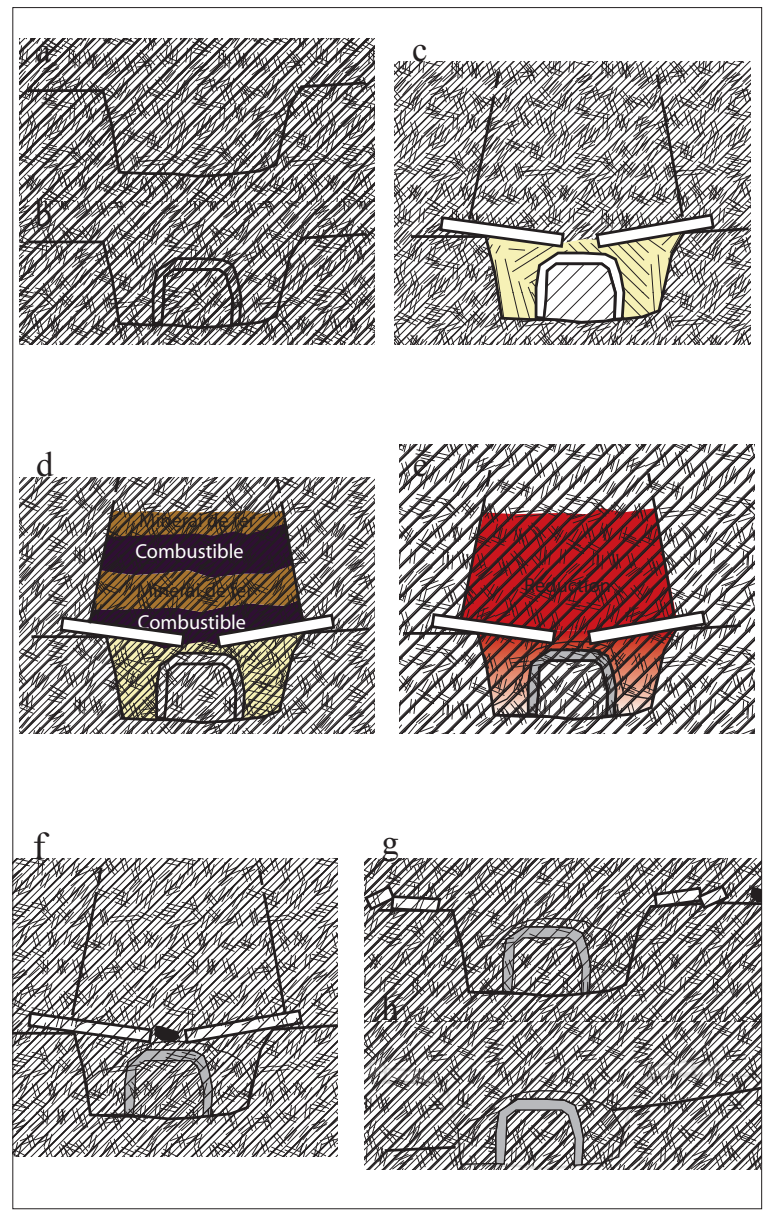

Figure 22. Hypothèse de fonctionnement des bas fourneaux à scorie piégée en anneau (SPa). a : Creusement d'une cuve circulaire (diam. $80 \mathrm{~cm}$ ) à fond plat et à paroi verticale ; b : Au centre, une motte de sable est construite (diam. $50 \mathrm{~cm}$ ), elle est tapissée d'argile sableuse (ep. 5-10 cm) ; c : La fosse de forme circulaire est remplie de paille de mil. Les tuyères sont disposées en appui sur le bord de la cuve et de l'îlot central. La cheminée est posée au-dessus, la jointure est assurée par du sable ; $d$ : Combustible et minerai de fer sont chargés par alternance par la cheminée. La réduction est déclenchée par le haut et est maintenue pendant plusieurs heures ; e : La scorie est piégée au fond de la cuve circulaire et recouvre l'îlot central ; $f$ : L'éponge de fer s'est formée entre les tuyères au-dessus de l'îlot; $g$ : Elle est récupérée après démontage de la cheminée; $\mathrm{h}:$ Le site est abandonné et soumis à l'érosion. Seul le bloc de scorie sous forme d'anneau ou d'une cloche est conservé.

former une colonne reliant le fond de la fosse aux orifices de la cuve. Enfin, l'action des métallurgistes lors de la construction ou d'intervention ponctuelle est déterminante sur la morphologie de la fosse et donc de la scorie qu'elle recueille. La diversité des bas fourneaux à scorie coulée serait alors issue de la capacité des métallurgistes à s'adapter au terrain sur lequel ils sont amenés à construire leurs bas fourneaux. Cette variabilité est également constatée au sein de leur organisation spatiale (GUILLON et al. 2013b).

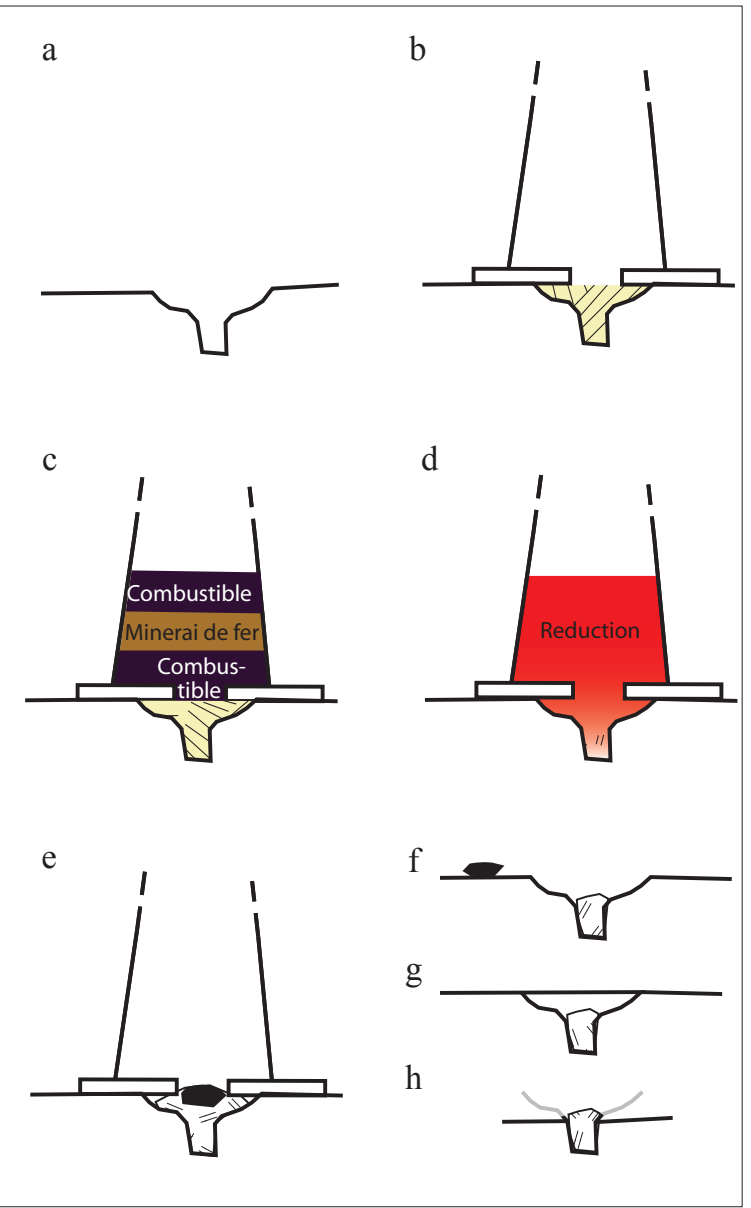

Figure 23. Hypothèse de fonctionnement des bas fourneaux à scorie piégée en cylindre $(\mathrm{SPc})$. a : Creusement d'une cuve (diam. $80 \mathrm{~cm}$ ) et d'une fosse (diam. 20-30 cm et prof. 20 $\mathrm{cm})$ au centre qui est rempli de paille de mil ; $b$ : Les tuyères sont disposées à plat en bordure de la cuve. Une plateforme est créée au dessus des tuyères pour recevoir la cheminée qui a été construite préalablement (ÉCHARD 1986) ; c : Combustible et minerai de fer sont chargés par alternance par la cheminée ; $d$ : La réduction est déclenchée par le haut et est maintenue pendant 24h environ (ÉcHARD 1986). La scorie est piégée au fond de la fosse; e : En fin de réduction, la scorie colmate la fosse. La loupe de fer s'est formée entre les becs des tuyères au dessus de la scorie ; $\mathrm{f}$ : La cheminée est déplacée pour récupérer la loupe de fer; g, h : Le bas fourneau est abandonné, la scorie est recouverte par le sable ou mise à nu si le sol est érodé.

Les bas fourneaux à scorie coulée à écoulement vertical sont rarement mentionnés, toutefois quelques éléments décrits par CÉLIS (1991) pour des bas fourneaux dit « à boule » dans la région de Tillabéry nous rappelleraient certaines caractéristiques des bas fourneaux SC-v sans que nous puissions réellement le vérifier. Des blocs massifs de scories cylindriques ont également été repérés au Nigéria sur les sites de Opi et de Leja, sans que leur mode de production n'ait été identifié (OKAFOR 1993, 2002 : 37). Cette technique semble avoir également été 


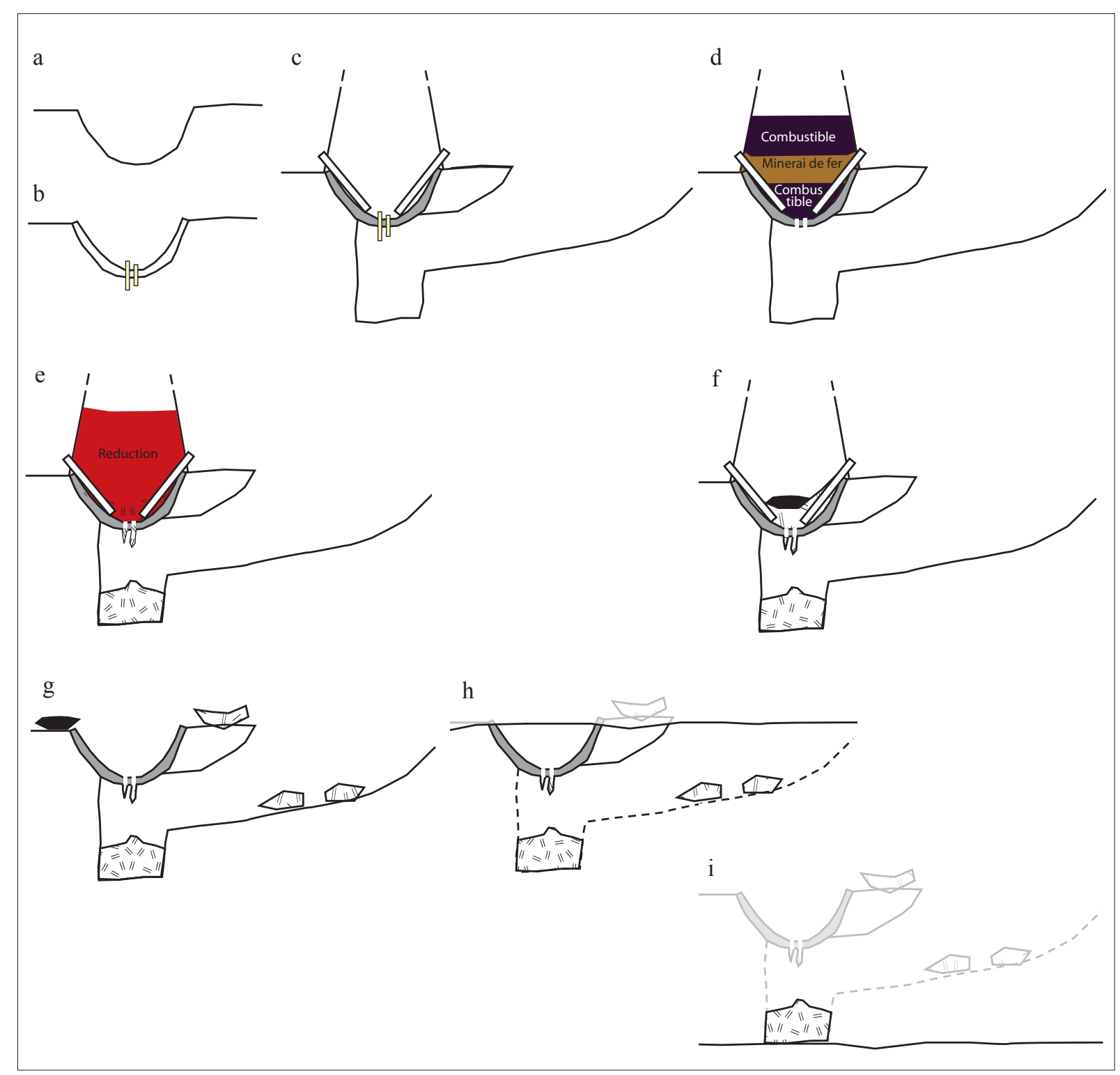

Figure 24. Hypothèse de fonctionnement des bas fourneaux à scorie coulée à écoulement vertical (SC-v). a : Creusement d'une cuve hémisphérique (diam. $100 \mathrm{~cm}$ ) qui est enduite d'une couche argilo-sableuse (ep. $5-10 \mathrm{~cm}) ; \mathrm{b}:$ Un orifice est perforé au fond de la cuve. Il est aussitôt obstrué par un bouchon consumable. La cuve est séchée ou précuite; $\mathrm{c}$ : Huit tuyères sont disposées à $45^{\circ}$ le long des parois de la cuve. La cheminée est posée au dessus des tuyères, la jointure est assurée par du sable. Une tranchée est ouverte au pied du bas fourneau pour réaliser une fosse sous la cuve en conservant des appuis suffisants ; $d$ : Combustible et minerai de fer sont chargés par alternance par la cheminée ; e : Le bouchon au fond de la cuve est éliminé manuellement ou détruit au contact de la scorie en fusion. Selon la température, la scorie s'écoule et forme sous la cuve des concrétions de goutelettes ou des drapées. À l'aplomb, la scorie s'accumule et forme un bloc dont la morphologie sera la contre empreinte de la fosse ; f: À la fin de la réduction, la scorie moins fluide peut former une colonne entre le point d'écoulement sous la cuve et le bloc de scorie dans la fosse. Dans la cuve, la scorie non écoulée se fige et colmate les perforations qui ont permis son écoulement; g : L'éponge de fer est récupérée après déplacement de la cheminée. Les débris sont en partie rejetés dans la tranchée. $\mathrm{h}, \mathrm{i}$ : Après abandon du site, si le sol est stable, seule le haut de la cuve reste visible, si le sol est fortement érodé, le bloc massif peut être découvert voire même démantelé.

observée au nord de Lagos au Nigéria à la fin du $19^{\mathrm{e}}$ siècle pour des bas fourneaux permanents. Ils sont construits sous un abri et constitués d'une cuve perforée d'un orifice de 7 à $10 \mathrm{~cm}$ qui communique avec un tunnel creusé sous le bas fourneau. Celui-ci permettait ainsi d'accéder au bouchon d'argile qui obstruait l'orifice et de veiller à ce que la scorie s'écoule correctement de la cuve (Bellamy 2009). Pour comprendre au mieux l'architecture du bas fourneau SC-v, nous proposons un mode de fonctionnement basé sur les relevés archéologiques (Fig. 24). 


\section{Continuité ou discontinuité ?}

Dans les environs de Niamey, la construction de ces quatre types de bas fourneaux semble s'interrompre à la fin du $14^{\mathrm{e}}$ siècle. La reprise de l'activité métallurgique n'est constatée qu'après le milieu du $17^{\mathrm{e}}$ siècle, ce que confirment les datations et les enquêtes menées auprès des populations. Or, selon les texte anciens et la tradition orale, à la fin de l'empire Songhaï, les « peuples anciens » abandonnent leur territoire vers le $16^{\mathrm{e}}$ et le $17^{\mathrm{e}}$ siècles et la région est investie par des strates d'immigrants plus ou moins anciens et hétéroclites entre le $16^{\mathrm{e}}$ et le $19^{\mathrm{e}}$ siècles (Olivier De SARDAN 1984 : 20). Ce changement de population dans la région concorde avec une forte baisse voire une absence d'activité métallurgique dans le Sud-ouest du Niger. L'arrêt de cette pratique a pu engendrer une perte de savoir-faire des métallurgistes ; alors qu'ils construisaient durant les siècles précédents plusieurs types de bas fourneaux à usage unique, seuls les bas fourneaux SPff à Banizoumbou et SPc à Saga Gorou semblent avoir été utilisés au-delà du $18^{\mathrm{e}}$ siècle. Il en est de même pour les bas fourneaux à usage multiple (BF-UM) découverts sur les plateaux de Louguel dont la datation mentionne une activité comprise entre le milieu du $17^{\mathrm{e}}$ et la fin du $18^{\mathrm{e}}$ siècles. Cette rupture d'activité entre la fin du $14^{\mathrm{e}}$ et le milieu du $17^{\mathrm{e}}$ siècles reste une hypothèse étant donné le risque d'un biais archéologique en l'absence de données chronologiques plus complètes. Cependant, cette période concorderait également avec l'abandon de grandes surfaces agricoles cultivées sur les plateaux de Louguel (GuILlon et al. 2013a) dont les talus et le glacis ont subi une intense érosion déchaussant un grand nombre de bas fourneaux (GUILLON 2013).

\section{Une production pour un marché local}

Le volume de la production en fer reste inconnu, mais celui des scories, les déchets, peut être évaluée en considérant le volume moyen d'une scorie proche de $0,1 \mathrm{~m}^{3}$. Le volume de scorie ne serait alors que d'environ $500 \mathrm{~m}^{3}$ dans les bassins versant de Saga Gorou, $160 \mathrm{~m}^{3}$ pour Banizoumbou, $260 \mathrm{~m}^{3}$ à Gobéri et au moins $100 \mathrm{~m}^{3}$ à Louguel. Ces données sont toutefois à pondérer avec la période de fonctionnement et l'aire d'influence de la production qui sont toutes les deux très délicates à évaluer. À Saga Gorou, d'après les 27 dates dont nous disposons, l'activité métallurgique s'étalerait sur 1000 ans, ce qui rapporterait le volume de production à $0,5 \mathrm{~m}^{3}$ par an soit la construction de cinq bas fourneaux par an. Bien que ce résultat relativise le volume de production de la région, il ne représente qu'une moyenne. À l'échelle d'un site sidérurgique comme celui de Bilfouda où la période d'activité est courte - la construction des 470 bas fourneaux de même type semble s'être étalée sur environ 25 ans — la production équivaut à $2 \mathrm{~m}^{3}$ par an soit 20 bas fourneaux par an, ce qui représente au plus 4 tonnes de scories par an. Même si le volume de production est plus élevé, il reste modeste et compatible avec une consommation locale. À Korsimoro au Burkina Faso, les traditions KRS1 et KRS4, toutes deux associées à des bas fourneaux à usage unique, génèrent une production annuelle de scorie inférieure à 100 tonnes (SERNEELs et al. 2014). Les bas fourneaux à usage unique ne peuvent concurrencer ceux construits de manière permanente comme dans la région de Bassar (DE BARRos 1986, 1988), du pays dogon (HUYSECOM et al. 2003 ; ROBION-BRUNNER 2010) ou de Korsimoro (KSR2 et KSR3) (SERnEELS et al. 2014) où la masse de scorie produite sur près de 1000 ans est respectivement d'environ un million, 800000 et 60000 tonnes. Au Sud-ouest du Niger, le fer semble donc être utilisé localement pour réaliser des parures et des outils pour l'artisanat, la pêche et la chasse. Même si aucun outil agricole n'a été collecté, l'aménagement et la mise en valeur des plateaux à proximité de l'ancien habitat de grande envergure de Louguel, et du site métallurgique qui compte quelques milliers de bas fourneaux témoignent très certainement de l'utilisation d'outils en fer (GuILlon et al. 2013b).

\section{Conclusion}

La présence de plusieurs types de bas fourneaux contemporains à usage unique sous-entend l'existence d'un savoir-faire varié. Les types de bas fourneaux ne semblent pas dépendants d'une zone géographique donnée, ils peuvent être en effet observés en différents lieux, sans que ces lieux ne soient spécifiques à un type de structure. Outre le signe d'une certaine mobilité des métallurgistes, la construction de ces types de bas fourneaux durant toute la période comprise entre 200 et 1400 cal AD témoignerait d'une transmission de savoir-faire sur de longues périodes sans que de véritables traditions ne se détachent comme à Korsimoro (SERnEels et al. 2012, 2014) ou au nord Bénin (ROBION-BRUNNER et al. 2015).

L'utilisation de bas fourneaux à usage unique semble l'indice d'une production de fer relativement limitée qui serait uniquement destinée à la population locale et non à un commerce de grande envergure. L'aire d'influence de ces productions paraît ainsi relativement réduite. La production de fer serait principalement destinée à la fabrication d'outils agricoles et d'armes de chasse.

Dans la région de Niamey, la déprise du territoire lors de la chute de l'empire Songhaï paraît perceptible par un hiatus archéologique qui est appuyé par des observations géomorphologiques attestant d'une intense érosion antérieure au $20^{\mathrm{e}}$ siècle. Cela conduit à être 
prudent sur l'usage des données ethnographiques pour les périodes antérieures au $15^{\mathrm{e}}$ siècle. Si l'ethnographie est nécessaire pour la compréhension des mythes et des rituels récents qui font partie intégrante des sociétés africaines, l'utilisation des données métallurgiques de ces sociétés contemporaines ne doit pas constituer un frein à l'interprétation des données archéologiques rendant compte du savoir-faire métallurgique des sociétés anciennes.

\section{Remerciements}

Nous remercions les relecteurs de cet article pour leurs nombreuses remarques constructives qui ont permis de clarifier et d'enrichir nos propos. Nous tenons à remercier tout particulièrement Alicot Maman, technicien à l'IRD Niger, pour son aide précieuse sur le terrain. Ce programme de recherche a été soutenu par le projet 6116 de la Coopération pour la Recherche Universitaire et Scientifique (CORUS2).

\section{Bibliographie}

Ackerman, K.J., Killick, D.J., Herbert, E.W. \& Kriger, C. 1999. A study of iron smelting at Lopanzo, Equateur Province, Zaïre. Journal of Archaeological Science 26 (8), 1135-1143. http://dx.doi.org/10.1006/jasc.1999.0409

Alpern, S.B. 2005. Did they or didn't they invent it? Iron in SubSaharan Africa. History in Africa 32, 41-94. http://dx.doi. org/10.1353/hia.2005.0003

de Barros, P. 1986. Bassar: a quantified, chronologically controlled, regional approach to a traditional iron production centre in West Africa. Africa 56 (2), 148-174. http://dx.doi. org/10.2307/1160630

de Barros, P. 1988. Societal repercussions of the rise of largescale traditional iron production: a West African example. The African Archaeological Review 6, 91-113. http://dx.doi. org/10.1007/BF01117114

Bellamy, C.V. 2009. Report of 1904 on traditional iron production. (A West African smelting house, Journal of the Iron and Steel Institute 66) Acta Archaeologica 80 (2), 42-49.

Bronk Ramsey, C. 2009. Bayesian analysis of radiocarbon dates. Radiocarbon 51 (1), 337-360.

Celis, G.R. 1987. Fondeurs et forgerons ekonda (Equateur, Zaïre). Anthropos 82, 109-134.

Celis, G.R. 1991. Les fonderies africaines du fer. Museum für Völkerkunde, Frankfurt am Main.

Celis, G.R. 1994. La métallurgie traditionnelle chez les Songhraï (Niger). Techniques et croyances : la reconstruction de Yelwani. Anthropos 89, 401-420.
Chièze V. 1991. La métallurgie du fer dans la zone lacustre. Archéologie et Archéométrie. In: Raimbault, M. \& Sanogo, $\mathrm{K}$. (eds), Recherches archéologiques au Mali: prospections et inventaire, fouilles et études analytiques en zone lacustre. Karthala Éditions, pp. 449-464.

Cleere, H.F. 1972. The classification of early iron-smelting furnaces. The Antiquaries Journal 52 (1), 8-23. http://dx.doi. org/10.1017/S0003581500020126

Cline, W. 1937. Mining and Metallurgy in Negro Africa, General Series in Anthropology. George Banta publishing company, Wisconsin, USA.

Clist, B. 2012. Vers une réduction des préjugés et la fonte des antagonismes: un bilan de l'expansion de la métallurgie du fer en Afrique sud-saharienne. Journal of African Archaeology 10 (1), 71-84. http://dx.doi.org/10.3213/2191-5784-10205

Échard, N. 1965. Note sur les forgerons de l'Ader (Pays Hausa, République du Niger). Journal de la Société des Africanistes 35 (2), 353-372.

Échard, N. 1968. Noces de Feu. 16 mm (20 min), Musée de l'Homme.

Échard, N. 1983. Scories et symboles, remarques sur la métallurgie hausa du fer au Niger. In: Échard, N. (ed), Métallurgies africaines. Nouvelles contributions. Mémoires de la société des africanistes 9. Société des africanistes, Paris, pp. 209-224.

Échard, N. 1986. Histoire du peuplement et histoire des techniques : l'exemple de la métallurgie hausa du fer au Niger. Journal des Africanistes 56 (1), 21-34.

Fabre, J.-M. 2009. La métallurgie du fer au Sahel burkinabé à la fin du 1er millénaire AD. In: Magnavita, S., Koté, L., Breunig, P. \& Idé, O. A. (eds), Crossroads / Carrefour Sahel. Cultural and Technological Developments in First Millennium BC/ AD West Africa. Journal of African Archaeology Monograph Series 2. Africa Magna, Frankfurt, pp. 167-178.

Fabre, J.-M. 2012a. Données archéologiques et ethnographiques : une confrontation parfois difficile. L'exemple du district sidérurgique de Markoye (Burkina Faso). In: Robion-Brunner, C. \& Martinelli, B. (eds), Métallurgie du fer et sociétés africaines. Bilans et nouveaux paradigmes dans la recherche anthropologique et archéologique. Cambridge Monographs in African Archaeology $\mathrm{N}^{\circ} 81$. BAR International Series 2395, Oxford, pp. 165-176.

Fabre, J.-M. 2012b. La vallée du Béli (Markoye, Burkina Faso) : un important district sidérurgique au temps des grands empires. In: Fauvelle-Aymar, F.-X. (ed), Palethnologie de l'Afrique. Palethnologie 4. Toulouse, pp. 195-209.

Francis-Bœuf, C. 1937. L'industrie autochtone du fer en Afrique Occidentale Française. Bulletin du comité d'études Historiques et scientifiques de l'AOF XX (4), 403-464.

Guillon, R., Petit, C., Rajot, J.L., Bichet, V., Idé O.A., Garba, Z., Sebag, D. \& Abdourhamane Touré, A. 2012. Analyse de la répartition spatiale de bas fourneaux d'un territoire sidérurgique au Sud-ouest du Niger $\left(4^{\mathrm{e}}-14^{\mathrm{e}}\right.$ siècle ap. J.-C.). In: Robion-Brunner, C. \& Martinelli, B. (eds), Métallurgie du fer et sociétés africaines. Bilans et nouveaux paradigmes dans 
la recherche anthropologique et archéologique. Cambridge Monographs in African Archaeology 81. BAR International Series 2395, Oxford, pp. 153-163.

Guillon, R. 2013. Relation société-milieu en domaine sahélien au Sud-ouest du Niger au cours des quatre derniers millénaires : approche géoarchéologique. Thèse non publiée, Université de Bourgogne, Dijon.

Guillon, R., Petit, C., Rajot, J.L., Abdourhamane Touré, A., Bichet, V., Garba, Z., Quiquérez, A. \& Sebag, D. 2013a. Stone lines and heaps on south-western Niger plateaus as remains of ancient agricultural land. In: Djindjian, F. \& Robert, S. (eds), Understanding Landscapes, from Land Discovery to their Spatial Organization. BAR International Series 2541, Oxford, pp. 87-96.

Guillon, R., Petit, C., Rajot, J.-L., Bichet, V., Sebag, D., Idé, O.A. \& Garba, Z. 2013b. Analyse de l'organisation spatiale de deux sites de production du fer dans le Sud-Ouest du Niger. ArchéoSciences. Revue d'archéométrie 37, 123-134.

Huysecom, E., Ballouche, A., Cissé, L., Gallay, A., Konaté, D., Mayor, A., Neumann, K., Ozainne, S., Perret, S., Raeli, F., Rasse, M., Robert, M., Robion, C., Sanogo, K., Serneels, V., Soriano, S. \& Stokes, S. 2003. Paléoenvironnement et peuplement humain en Afrique de l'ouest : rapport de la sixième campagne de recherche à Ounjougou (Mali), Jahresbericht SLSA, 27-68.

Kiénon-Kaboré, T.H. 2005. Métallurgie Ancienne du fer au Burkina Faso Province du Bulkiemdé : approche ethnologique, historique, archéologique et métallographique. L'Harmattan, Paris.

Kiéthéga, J.B. 2006. La métallurgie lourde du fer au Burkina Faso: Une technologie à l'époque précoloniale. Karthala Éditions, Paris.

Killick, D. 2015. Iron smelting technology in the middle Senegal Valley, ca. 550 BCE - 1500 BCE. In: McIntosh R. J., McIntosh, S.K. \& Bocoum, H., The Search for Takur : Archaeological Excavations and Reconnaissance along the Middle Senegal Valley. Yale University Press, New Haven (sous presse).

Leroy, M. 2001. La sidérurgie en Lorraine avant le haut fourneau. L'utilisation du minerai de fer oolithique en réduction directe. Monographie du CRA, CNRS Éditions, Paris.

Martinelli, B. 1993. Fonderies ouest-africaines. Classement comparatif et tendances. Techniques et Culture 21, 195-221.

McIntosh, S.K. \& Bocoum, H. 2000. New perspectives on Sincu Bara, a first millennium site in the Senegal valley African Archaeological Review 17 (1). 1-43. http://dx.doi. org/10.1023/A:1006694511823

McNaughton, P.R. 1993. The Mande Blacksmiths: Knowledge, Power, and Art in West Africa. Indiana University Press. Bloomington and Indianapolis.

Okafor, E.E. 1993. New evidence on early iron-smelting from southeastern Nigeria. In: Shaw, T., Sinclair, P., Andah, B. \& Okpoko, A. (eds), The Archaeology of Africa. Food, Metals and Towns. One World Archaeology 20. Routledge, London, pp. $432-448$
Okafor, E.E. 2002. La réduction du fer dans les bas fourneaux, une industrie vielle de 2500 ans au Nigéria In: Bocoum, H. (ed), Aux origines de la métallurgie du fer en Afrique : une ancienneté méconnue Afrique de l'Ouest et Afrique centrale. Mémoires des Peuples, UNESCO, Paris, pp. 35-48.

Olivier de Sardan, J.-P. 1984. Les Sociétés songhay-zarma (Niger-Mali). Chefs, guerriers, esclaves, paysans..., Karthala Editions, Paris.

Pelet, P.-L. 1993. Une industrie reconnue: fer, charbon, acier dans le Pays de Vaud. Cahiers d'archéologie romande 60, Lausanne.

Pleiner, R. 2000. Iron in Archaeology: The European Bloomery Smelters. Archeologickýústav AVČR.

Pole, L.M. 1985. Furnace design and the smelting operations. In: Haaland, R. \& Shinnie, P.L. (eds), African Iron Working. Norwegian University Press, Olso, pp. 142-163.

Robert-Chaleix, D. 1994. Métallurgie du fer dans la moyenne vallée du Sénégal : les bas fourneaux de Silla. Journal des Africanistes 64 (2), 113-127.

Robert-Chaleix, D. \& Sognane, M. 1983. Une industrie métallurgique ancienne sur la rive mauritanienne du fleuve Sénégal. In: Échard, N. (éd), Métallurgies africaines. Nouvelles contributions. Mémoire de la société des africanistes 9. Société des africanistes, Paris, pp. 45-62.

Robion-Brunner, C. 2010. Forgerons et sidérurgie en pays dogon. Vers une histoire de la production du fer sur le plateau de Bandiagara (Mali) durant les empires précoloniaux. Journal of African Archaeology Monograph Series 3. Africa Magna, Frankfurt.

Robion-Brunner, C., Haour, A., Coustures, M.P., Champion, L. \& Béziat, D. 2015. Iron production in Northern Benin: Excavation at Kompa Moussékoubou. Journal of African Archaeology 13 (1), 39-57.

Serneels, V. 1993. Archéométrie des scories de fer: recherches sur la sidérurgie ancienne en Suisse occidentale.Cahiers d'archéologie romande 61, Lausanne.

Serneels, V. 1998. La chaine opératoire de la sidérurgie ancienne. In: Feugère, M. \& Serneels, V. (eds), Recherches sur l'économie du fer en Méditerranée nord-occidentale. Monographies intrumentum 4. Éditions Monique Mergoil, pp. 7-44.

Serneels, V., Donadini, F., Kiénon-Kaboré, T.H., Koté, L., Kouassi, K.S., Ramseyer, D. \& Simporé, L. 2014. Origine et développement de la métallurgie du fer au Burkina Faso et en Côte d'Ivoire. Avancement des recherches en 2013 et quantification des vestiges de Korsimoro (Burkina Faso). Jahresbericht SLSA 2013, 65-112.

Serneels, V., Kiénon-Kaboré, T.H., Koté, L., Kouassi, K.S., Ramseyer, D. \& Simporé, L. 2012. Origine et développement de la métallurgie du fer au Burkina Faso et en Côte d'Ivoire. Premiers résultats sur le site sidérurgique de Korsimoro (Sanmatenga, Burkina Faso). Jahresbericht SLSA 2011, 23-54.

Sutton, J.E.G. 1985. Temporal and spatial variability in African iron furnaces. In: Haaland, R. \& Shinnie, P. L. (eds), African Iron Working. Norwegian University Press, Oslo, pp. 164-191. 


\section{not cited in text}

Tylecote, R.F. 1975. Iron-smelting at Taruga, Nigeria. Journal of Historial Metallurgy Society 9 (2), 49-56.

Tylecote, R.F. 1987. The Early History of Metallurgy in Europe. Longman Archaeology Series, London, New York.

Vernet, R. 1996. Le sud ouest du Niger: de la Préhistoire au début de l'histoire. Études Nigériennes 56. IRSH, SEPIA, Niamey, Paris. 